\title{
DESIGNING MULTI-PERIOD SUPPLY CONTRACTS IN A TWO-ECHELON SUPPLY CHAIN WITH ASYMMETRIC INFORMATION
}

\author{
Zahra Mobini, Wilco van den Heuvel, Albert Wagelmans
}

Econometric Institute Report 2014-28

\begin{abstract}
We study a two-echelon supply chain consisting of a supplier and a retailer, where the supplier uses a simple and easily implementable incentive scheme - making a side payment - to influence the retailer's ordering plan. The supplier makes a take-it-or-leave-it offer to the retailer in the form of a menu of contracts, each consisting of a procurement plan plus a side payment. The retailer, who possesses private information about customer demand and his cost parameters, either accepts one of the contracts or imposes his own optimal plan. We formulate the supplier's problem of designing optimal contracts with the realistic assumption that the retailer's outside option depends on his private information. Taking into account the retailer's reaction to the proposed offer, the supplier faces a nested (bi-level) optimization problem, which we transform into a single-level mixed integer programming formulation. In our analysis, we use a network interpretation for the set of incentive constraints and show some properties of optimal contracts. This enables us to considerably reduce the number of incentive constraints and to find optimal values of the side payment quantities. Our findings regarding the possible behavior of the opportunistic retailer deviate from those of previous studies as a result of considering more realistic assumptions.
\end{abstract}

Keywords Supply chain contracting, coordination mechanisms, lot-sizing, asymmetric information.

\section{INTRODUCTION}

A supply chain (SC) generally consists of separate, individual members with different preferences and objectives, making decisions autonomously and striving to meet their own local objectives. Such selfish, opportunistic behavior can lead to suboptimal performance of the whole SC. The loss of efficiency because of the opportunistic behavior of SC members is referred to as the "price of anarchy" in the literature (Perakis \& Roels 2007).

To overcome SC inefficiencies and reduce the price of anarchy, a single, central decision-maker in a so-called centralized SC can optimize overall SC performance, e.g., maximize the profits realized at each level of the SC by fulfilling customer demand. This solution is, however, not without problems. First, the centralized solution may not necessarily be in the best interest of every individual SC member; some members may incur significant losses, while others may earn substantial profits. As a result, autonomous members may seek to meet their own individual objectives, rather than those of the entire SC system. Second, to be able to fully integrate and control the SC system, the central decision-maker needs to have access to all SC information, such as the market demand for the final product, inventory level, and cost/profit structure of each individual member. Access to such comprehensive information rarely happens in reality, and it is likely that no two members possess comparable information. One member may have a better forecast of demand than another, or a member may possess superior information regarding the costs and operating procedures of other members. Third, the idea of a centralized SC 
system being controlled by only a single decision-maker is not a viable option because it is usually costly and carries noticeable risks, in addition to being difficult to administer.

Given the decentralized nature and existence of information asymmetry (IA) in most real-world supply chains, it is important to coordinate decisions and activities of individual SC members in order to improve the performance of the whole SC and reduce the price of anarchy. This paper considers a typical two-echelon SC, consisting of a supplier who serves a retailer with a single product. The retailer faces an economic lot sizing (ELS) problem to find an ordering plan that maximizes his total profit of satisfying customer demand. The supplier also faces an ELS problem to find a production plan that maximizes the total profit earned by fulfilling the retailer's orders.

In our SC, the retailer has the market power to impose his optimal ordering plan on the supplier. Large and financially successful retailers, such as Wal-Mart, whose large-share suppliers depend on the retailer's broad market reach to maintain their sales volumes and strengthen their market positions (Bloom \& Perry 2001), constitute real-world examples of such an imbalanced power relationship.

To persuade the strong retailer to choose an ordering plan that accords with the supplier's cost structure, rather than the retailer's individual optimal plan, the supplier can pay a side payment to the retailer. To determine a side payment sufficient to influence the retailer's behavior, the supplier needs to know the cost/profit function and external demand at the retailer's end. In general, the supplier does not have access to such information about the retailer, and the retailer may use his private information strategically.

In this paper, we formulate the problem of designing optimal contracts from the supplier's perspective under three conditions: (a) no IA, (b) IA across one dimension (the retailer's cost type), and (c) IA across two dimensions (the retailer's cost(s) type and customer demand). Although the individual SC members make their procurement decisions independently of each other, the profit they realize depends not only on their own decisions, but also on the decisions of the other SC members. Such a separate, interactive decision-making approach requires the supplier to take into account the retailer's reaction to his proposed offer. This corresponds to a nested (bi-level) optimization problem for the supplier, which we transform into a single-level Mixed Integer Programming (MIP) model.

In the analysis of models with IA, we use a natural network interpretation for the set of incentive compatibility constraints and show some properties of optimal contracts. This allows us to considerably simplify the set of incentive compatibility constraints; rather than working through a large set of constraints, we can focus our attention on a more tractable set. Additionally, to find the optimal side payment quantities we only need to consider constraints that correspond to paths in the relevant network.

An important feature of our study is the dependence between the retailer's outside option (Muthoo 1999) and his private information. That is, we drop the simplifying assumption, prevalent in the mechanism design literature, that retailer types with different private information share an identical reservation profit. This departure from the previous literature is particularly important as we also consider more than one dimension of IA and a discrete type space for each dimension. Our more realistic assumption together with the considered IA setting 
enables us to present unexpected results with respect to the possible behavior of the opportunistic retailer.

An interesting property of the optimal solution in the one-dimensional setting corresponds to a well-known result in the mechanism design literature that an inefficient type (e.g., the one with the higher cost) does not pretend to be efficient (e.g., lower cost) in order to gain a larger part of the efficiency gain. We, however, show that the information rents are not necessarily monotonic and therefore the inefficient type may make profit by pretending to be efficient.

In the two-dimensional cost uncertainty case and considering a binary type space for each dimension with equal probability for each type, we show that a type that is inefficient in two dimensions (e.g., high set-up and holding cost) may pretend to be efficient in both dimensions and the other way around. It is, however, not profitable for a low set-up cost and high holding cost to pretend to have high set-up and low holding cost and vice versa. It was previously shown in the literature that a retailer who is strong in one dimension (e.g., high demand) but weak (e.g., high cost) in the other would sometimes prefer a situation where his weak dimension is known to the supplier to a situation where both dimensions are private information. With an example, we demonstrate that it might be in the interest of a retailer type who is strong in both dimensions that one of the dimensions is known to the supplier.

The remainder of this paper is organized as follows. First, Section 2 provides a review of the relevant SC coordination literature. Next, we present a sequence of models, starting from the simple one, and getting more complex as we proceed. The model for deriving the individual optimal procurement plan of the two SC members is discussed in Section 3. Section 4 analyzes the SC optimal procurement plan from the viewpoint of a central decision-maker. The model for the decentralized SC system, in which there is no IA between the retailer and supplier, is discussed in Section 5. The formulation and network interpretation of the supplier's problem of designing optimal contract menus with one and two dimensions of IA is discussed in Sections 6 and 7 , respectively. The paper ends with a conclusion in Section 8 .

\section{Literature REVIEW}

Although there is a vast body of literature on coordination of different SC settings, we restrict our review to some of the relevant studies, dealing with the coordination problem with IA. To classify the prior research on SC coordination, a distinction is made based on the nature of customer demand: deterministic and constant, deterministic but dynamic, and stochastic demand.

The focus of one strand of research in SC coordination is on the economic order quantity (EOQ) setting where demand and cost parameters are independent of time and the time horizon has a continuous scale of infinite length. Corbett \& de Groote (2000) developed a screening model with IA on the buyer's holding cost which has a continuous type space. The supplier, who follows a lot-for-lot policy, offers a menu of price-quantity contracts to maximize his own profit. Sucky (2006) argued that the problem of IA in this model is actually eliminated as the supplier can derive the buyer's holding cost by knowing his EOQ and ordering cost. Sucky (2006) presented a screening model with IA on both the holding and ordering costs of the buyer wherein the reservation values 
of the two buyer types are not identical. The seller pays a side payment to influence the buyer's ordering plan.

Another class of research in a deterministic but non-stationary demand environment uses the ELS model as the basic model for coordination. Dudek \& Stadtler (2005) developed a negotiation-based model for synchronizing plans between a buyer and a supplier in which both SC members use MIP models to generate their offers. Later, the same authors extended this work in Dudek \& Stadtler (2007) by considering a SC consisting of one supplier and several buyers who face a capacitated lot-sizing model to generate their offers and the amount of information exchanged between parties is further reduced.

There are also some studies in the class of stochastic models, addressing the SC coordination problem. Ha (2001) used the setting of the newsvendor model, where demand is stochastic and price sensitive. The author provided a screening model where the supplier does not know the buyer's marginal cost and assumes a continuous type space for the marginal cost. The paper showed that it is not possible to implement an optimal solution of the complete information case. In a single-period setting, Özer \& Wei (2006) analyzed the problem of credible information sharing between a supplier and a manufacturer who possesses private forecast information of her end product. Considering a continuous type space for demand forecast, they showed that an appropriate payback contract can coordinate the SC. In a similar setting, i.e., one-period planning horizon and IA about demand forecast, Taylor (2006) examined the effect of the retailer's private information on the manufacturer's sale-timing decision. It was shown that the manufacturer, who offers a price-quantity contract for each element of a discrete demand type space, may prefer to sell close to the selling season if his production cost is low. Kostamis \& Duenyas (2011) studied a SC consisting of a supplier and a manufacturer who has private information over two dimensions of market demand and his in-house production cost. The authors concluded that with a screening game with two uncertain dimensions, the SC performance may deteriorate by sharing information. A SC with a retailer and a supplier who has private information about his unit production cost was analyzed in Çakanyıldırım et al. (2012). The retailer offers a menu of contracts to the supplier with the assumption that the outside opportunities of the two different types are not identical. The paper concluded that IA alone does not necessarily induce loss in channel efficiency.

Table 1 summarizes the literature discussed in this section and positions our paper. Although these studies incorporate various aspects of SC coordination, their focuses are mainly on the design and analysis of mechanisms in settings with tightly defined assumptions. Corbett \& de Groote (2000) in the EOQ setting, Ha (2001) and Çakanyıldırım et al. (2012) in a single-period stochastic environment considered one-dimensional private information about a cost parameter. IA about demand forecast in a single-period setting was analyzed by Özer \& Wei (2006) and Taylor (2006). Assuming a two-dimensional setting, Sucky (2006) studied a coordination problem with two uncertain cost parameters, while Kostamis \& Duenyas (2011) dealt with uncertain market demand and a cost parameter. The underlying multi-period inventory model in Dudek \& Stadtler $(2005,2007)$ is similar to ours, but our proposed mechanism is simpler and seems easier to implement than the iterative, negotiation-like scheme suggested in these papers. Additionally, their negotiation process does not completely 
rule out the possibility of cheating by the buyers.

In contrast to much of the literature in which identical outside options for different retailer's types are assumed, we consider the case where the retailer's outside option depends on his private information. Although Sucky (2006) and Çakanylldırım et al. (2012) did not assume a common outside option for different types, both studies focused on settings where each dimension of private information has a binary type space (i.e., low or high). We consider a more general setting with a discrete type space for each dimension.

Table 1: Classification of supply chain coordination models with information asymmetry

\begin{tabular}{|c|c|c|c|c|c|c|c|}
\hline & & & One-period & EOQ & ELS & IA setting & Type space \\
\hline \multirow{5}{*}{ 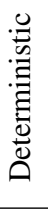 } & \multirow{5}{*}{ 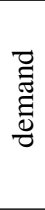 } & Corbett \& de Groote (2000) & & $\checkmark$ & & $1 \mathrm{dim}$. & Continuous \\
\hline & & Sucky (2006) & & $\checkmark$ & & $2 \mathrm{dim}$. & Binary \\
\hline & & Dudek \& Stadtler (2005) & & & $\checkmark$ & NA* & NA* \\
\hline & & Dudek \& Stadtler (2007) & & & $\checkmark$ & NA* & NA* \\
\hline & & This paper & & & $\checkmark$ & $2 \mathrm{dim}$. & Discrete \\
\hline \multirow{5}{*}{ 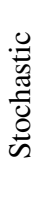 } & \multirow{5}{*}{ 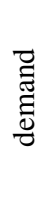 } & Нa (2001) & $\checkmark$ & & & $1 \mathrm{dim}$. & Continuous \\
\hline & & Özer \& Wei (2006) & $\checkmark$ & & & $1 \mathrm{dim}$. & Continuous \\
\hline & & Taylor (2006) & $\checkmark$ & & & $1 \mathrm{dim}$. & Discrete \\
\hline & & Kostamis \& Duenyas (2011) & $\checkmark$ & & & $2 \mathrm{dim}$. & Binary \\
\hline & & Çakanyıldırım et al. (2012) & $\checkmark$ & & & $1 \mathrm{dim}$. & Binary \\
\hline
\end{tabular}

* Dudek \& Stadtler $(2005,2007)$ do not fit into these categories as they did not use the mechanism design approach.

\section{INDIVIDUAL OPTIMAL PROCUREMENT PLANS}

In this paper, we restrict our analysis to a two-echelon serial SC consisting of a risk-neutral supplier who serves a risk-neutral retailer with a single product. The retailer faces an ELS problem to find an ordering plan that maximizes his total profit of satisfying external customer demand. The supplier also faces an ELS problem to find a production plan that maximizes the total profit earned by fulfilling the retailer's orders.

The ELS model determines the optimal timing and procurement (the term "procurement" is used to cover both ordering -at the retailer's level- and production -at the supplier's level- settings) level in order to satisfy a deterministic but dynamic demand over a discrete time horizon of finite length. The model can be described as follows. For a fixed planning horizon of length $T$, there is a known demand for a single item. This demand has to be satisfied each period by procurement in this period or in previous periods, i.e., backlogging is not allowed. Relevant costs include procurement costs and holding (inventory) costs. The procurement costs consist of a fixed component (e.g., set-up cost) and marginal procurement cost per item. We use the following notation for the parameters in periods $t \in \mathcal{T}=\{1,2, \ldots, T\}$ :

$$
\begin{aligned}
& d_{t}: \text { demand in period } t \\
& K_{t}: \text { fixed procurement cost in period } t \\
& p_{t}: \text { unit variable procurement cost in period } t \\
& h_{t}: \text { unit holding cost in period } t \\
& s_{t}: \text { unit selling price in period } t .
\end{aligned}
$$

The objective is to determine how much to procure in each period such that demand is fully met at maximum profit. There are three decision variables for periods $t \in \mathcal{T}=\{1,2, \ldots, T\}$ : 
$x_{t}$ : procurement quantity in period $t$

$y_{t}$ : set-up variable in period $t, 1$ if there is any procurement and 0 otherwise

$I_{t}$ : ending inventory in period $t$.

The basic single-item, uncapacitated ELS problem can be formulated as follows:

$$
\begin{array}{rr}
\max \sum_{t=1}^{T}\left(s_{t} d_{t}-K_{t} y_{t}-p_{t} x_{t}-h_{t} I_{t}\right) \\
\text { s.t. } \quad I_{t-1}+x_{t}=I_{t}+d_{t} & \forall t \in \mathcal{T} \\
x_{t} \leq y_{t} d_{t, T} & \forall t \in \mathcal{T} \\
x_{t}, I_{t} \geq 0 & \forall t \in \mathcal{T} \\
y_{t} \in\{0,1\} & \forall t \in \mathcal{T} \\
I_{0}=0 &
\end{array}
$$

The objective is to maximize profit earned by selling the product minus procurement and inventory holding costs. We assume that the unit selling price of the supplier's profit in (1) is equal to the retailer's unit variable procurement cost. Demand can be satisfied from procurement in the current period or inventory left over from previous periods, while remaining items build up the current inventory. This is formalized in (2). Constraints (3) are the set-up forcing constraints, which together with constraints (5) ensure that the set-up variable is forced to 1 if there is any procurement. As there is no ending inventory in the last period of the optimal solution and costs are assumed to be non-negative, procurement is limited by the remaining cumulative demand. Note that in this formulation, $d_{s, t}=\sum_{i=s}^{t} d_{i}$ denotes the cumulative demand from period $s$ up to period $t$ with $1 \leq s \leq t \leq T$. Constraints (4) ensure that the procurement quantity and ending inventory are non-negative. Finally, the starting inventory is assumed to be zero (6).

If the supplier and retailer behave individually rational, they each use the above formulation to maximize their own profits. These locally optimal plans, however, are not efficient from the system-wide perspective and both parties can benefit if their procurement decisions are coordinated. We illustrate the ELS problem by an example.

Example 1. Consider that the retailer faces a 5-period ELS problem with the parameters according to Table 2 a. Further assume that the retailer has the market power to impose his individual optimal plan on the supplier. The optimal solution of the retailer's problem is to order 26 units in period 1,25 units in period 2 , and 73 units in period 3 with the total profit of 466 units. Now this optimal ordering plan becomes the demand for the supplier. Table $2 \mathrm{~b}$ lists the parameters of the supplier's problem. The supplier's optimal production plan is to produce 51 units in period 1 and 73 units in period 3, with the total profit of 286 units. The total SC profit equals $752(=466+286)$ units. 
Table 2: Parameters of the ELS problem

(a) Parameters of the retailer's problem

\begin{tabular}{cccccc}
\hline$t$ & 1 & 2 & 3 & 4 & 5 \\
\hline \hline$d_{t}$ & 26 & 25 & 29 & 33 & 11 \\
$K_{t}$ & 179 & 199 & 282 & 285 & 292 \\
$p_{t}$ & 15 & 17 & 11 & 15 & 15 \\
$h_{t}$ & 10 & 16 & 10 & 15 & 13 \\
$s_{t}$ & 30 & 21 & 28 & 29 & 25
\end{tabular}

(b) Parameters of the supplier's problem

\begin{tabular}{cccccc}
\hline$t$ & 1 & 2 & 3 & 4 & 5 \\
\hline \hline$d_{t}$ & 26 & 25 & 73 & 0 & 0 \\
$K_{t}$ & 327 & 280 & 434 & 450 & 338 \\
$p_{t}$ & 2 & 1 & 3 & 4 & 3 \\
$h_{t}$ & 10 & 18 & 11 & 15 & 14 \\
$s_{t}$ & 15 & 17 & 11 & 15 & 15 \\
\hline
\end{tabular}

\section{SUPPLY CHAIN OPTIMAL PROCUREMENT PLAN}

To provide a basis for comparison, in this section, we discuss the model for the ideal case of the centralized SC. In the centralized decision-making setting, a single decision-maker with access to all the required information aims to determine the joint supplier-retailer procurement plan that maximizes total SC profits. The profit maximization problem of our SC can be formulated as follows:

$$
\begin{aligned}
& \max \sum_{t=1}^{T}\left[\left(p_{t}^{R} x_{t}^{R}-K_{t}^{S} y_{t}^{S}-p_{t}^{S} x_{t}^{S}-h_{t}^{S} I_{t}^{S}\right)+\left(s_{t}^{R} d_{t}-K_{t}^{R} y_{t}^{R}-p_{t}^{R} x_{t}^{R}-h_{t}^{R} I_{t}^{R}\right)\right] \\
& \text { s.t. } \quad I_{t-1}^{S}-I_{t}^{S}+x_{t}^{S}=x_{t}^{R} \forall t \in \mathcal{T} \\
& I_{t-1}^{R}-I_{t}^{R}+x_{t}^{R}=d_{t} \forall t \in \mathcal{T} \\
& x_{t}^{S} \leq d_{t, T} y_{t}^{S} \forall t \in \mathcal{T} \\
& x_{t}^{R} \leq d_{t, T} y_{t}^{R} \forall t \in \mathcal{T} \\
& x_{t}^{R}, x_{t}^{S}, I_{t}^{R}, I_{t}^{S} \geq 0 \forall t \in \mathcal{T} \\
& y_{t}^{R}, y_{t}^{S} \in\{0,1\} \forall t \in \mathcal{T} \\
& I_{0}^{R}, I_{0}^{S}=0
\end{aligned}
$$

In this formulation, the decision variables are divided into two separate sets: supplier $(S)$ and retailer $(R)$. Based on customer demand, the retailer places an order with the supplier and then, the retailer's order becomes the demand $\left\{x_{t}^{R}\right\}_{t \in \mathcal{T}}$ for the supplier. The inventory balance constraints of the supplier and the retailer are formalized in (8) and (9), respectively. Constraints (10) and (11) are the set-up forcing constraints. Note that in constraint (10), the cumulative customer demand of the retailer is considered as an upper bound for the retailer's order quantity, which in turn constitutes the demand for the supplier. The objective is to maximize the joint ordering-production profits at two levels of the SC (7). Zangwill (1969) proposed an efficient dynamic programming algorithm to solve the cost minimization version of this problem.

Definition 2. We define the efficiency of a SC system under a particular coordination mechanism to be the fraction by which the gap between the profit of the centralized $\left(\pi^{C e n t}\right)$ and sequential $\left(\pi^{S e q}\right)$ decision-making settings is reduced. That is, the coordination mechanism efficiency is defined as the difference between the profit of the coordinated $\left(\pi^{C o}\right)$ and sequential setting solutions relative to the difference of the profit associated with the centralized and sequential setting solutions:

$$
\text { Coordination mechanism efficiency }=\frac{\pi^{C o}-\pi^{S e q}}{\pi^{C e n t}-\pi^{S e q}} \text {. }
$$


We use this measure in the subsequent sections to quantify the SC profit improvement, resulting from the implementation of a coordination mechanism.

Example 3. Using the parameters in Table 2, we show the performance of the SC system in the centralized setting. The optimal plan for both the retailer and supplier is to procure 51 units in period 1 and 73 units in period 3 with the profit of 465 and 486 units, respectively. The total SC profit is 951 units.

\section{OPTIMAL CONTRACT UNDER SYMMETRIC INFORMATION}

To capture a more realistic situation, the focus in this section shifts from the centralized setting to the decentralized SC, where the supplier is in charge of designing a take-it-or-leave-it contract. The assumption here is that both the supplier and the retailer are in the same position with respect to the information at the retailer's end. This means that the supplier knows demand and cost type of the retailer. He can then incorporate this information into his optimization problem to come up with the procurement plan and the required side payment in order to influence the retailer's ordering plan.

The sequence of events is as follows. The supplier and retailer observe customer demand. Knowing the retailer's cost type, the supplier can derive the maximum profit that the retailer will earn by satisfying customer demand. This profit is denoted by $\pi^{R *}$ in our model. Based on customer demand, the retailer's optimal profit $\left(\pi^{R *}\right)$ and his own cost parameters, the supplier determines his production plan and side payment quantity such that his profit is maximized. Subsequently, the supplier makes an offer to the retailer in the form of a contract, consisting of a procurement plan plus a side payment. The retailer either accepts the offer or imposes his optimal ordering plan on the supplier. The offered contracts in our study have a stationary format. That is, the procurement and side payment quantities, once determined, do not change throughout the planning horizon. In addition to providing tractability to the model analysis, this assumption is of particular interest to our problem setting for several practical reasons. First, contracts with time-varying parameters would be unnecessarily complex, making them more difficult to implement in practice. Second, modifying contracting terms throughout the planning horizon may be costly as it may require renegotiations between the SC members and cause planning problems (see Zhang (2010) for more discussions). Third, our model can be valuable for future research by serving as an initial step to understand the problem of designing supply contracts with more realistic assumptions.

The total profit that the retailer will realize by satisfying customer demand is denoted by $\pi^{R}$. If the retailer chooses the procurement plan-side payment pair $\left\{\left(x_{t}^{R}\right)_{t \in \mathcal{T}}, z\right\}$ from the supplier's contract, his total profit is given by $\pi^{R}+z=\sum_{t=1}^{T}\left(s_{t}^{R} d_{t}-K_{t}^{R} y_{t}^{R}-p_{t}^{R} x_{t}^{R}-h_{t}^{R} I_{t}^{R}\right)+z$ where $z$ is the quantity of side payment. The supplier needs to ensure that the offered contract is individually rational, i.e., he must guarantee that by accepting the offered contract, the retailer will earn a profit (weakly) larger than his "outside option". The retailer's outside option in our model is the optimal profit of his individual ordering plan.

The supplier's design of the optimal contract can be formulated as follows:

$$
\max \sum_{t=1}^{T}\left(p_{t}^{R} x_{t}^{R}-K_{t}^{S} y_{t}^{S}-p_{t}^{S} x_{t}^{S}-h_{t}^{S} I_{t}^{S}\right)-z
$$




$$
\begin{array}{rlrl}
\text { s.t. } & \pi^{R}+z \geq \pi^{R *} & \\
\pi^{R}=\sum_{t=1}^{T}\left(s_{t}^{R} d_{t}-K_{t}^{R} y_{t}^{R}-p_{t}^{R} x_{t}^{R}-h_{t}^{R} I_{t}^{R}\right) & \\
I_{t-1}^{R}+x_{t}^{R}=d_{t}+I_{t}^{R} & \forall t \in \mathcal{T} \\
x_{t}^{R} \leq d_{t, T} y_{t}^{R} & \forall t \in \mathcal{T} \\
I_{t-1}^{S}+x_{t}^{S}=x_{t}^{R}+I_{t}^{S} & \forall t \in \mathcal{T} \\
x_{t}^{S} \leq d_{t, T} y_{t}^{S} & \forall t \in \mathcal{T} \\
z \geq 0 & & \\
x_{t}^{R}, x_{t}^{S}, I_{t}^{R}, I_{t}^{S} \geq 0 & \forall t \in \mathcal{T} \\
y_{t}^{S}, y_{t}^{R} \in\{0,1\} & \forall t \in \mathcal{T} \\
I_{0}^{R}, I_{0}^{S}=0 &
\end{array}
$$

Constraint (16) is the "individual rationality" (IR) or participation constraint to ensure that by accepting the supplier's offer the retailer's gain is at least his outside option. The constraints of the retailer's individual ELS problem are formalized in (18) and (19), while those of the supplier are presented in (20) and (21). Constraint (22) ensures that the side payment quantity is non-negative and constraints (23) to (25) define the range of other decision variables.

To illustrate that the described problem has a feasible solution, consider the case where $z=0$. This implies that the supplier will not pay any side payment to the retailer to influence his individual optimal plan. The solution of the model in this situation is the outcome of the decentralized (uncoordinated) SC, where the retailer imposes his outside option on the supplier and the supplier is forced to satisfy the retailer's individual optimal plan.

Observe that we have a maximization problem in which the optimal choice of $z$ would be to set it as small as possible. In this case, constraint (16) would be binding and therefore the supplier maximizes not only his own profit, but also the profit of the SC system as a whole. By designing the contract in this manner, the supplier ensures that the retailer earns his individual optimal profit and extracts all the efficiency gains for himself. This solution is known as the "first-best" solution. With IA, however, SC optimal profit may not be achieved as shown in the subsequent sections.

Example 4. Using the parameters in Table 2, the contract that the supplier offers consists of procuring 51 units in period 1 and 73 units in period 3 plus 1 unit of side payment. The retailer's profit equals $466(=465+1)$. The optimal plan of the supplier is the same as the retailer's plan with the total profit of 485 units, and the total SC profit would be 951 units. As expected, the total SC profit is the same as the profit of the centralized setting (Example 3) and therefore the efficiency of the coordination mechanism (making a side payment) is 1. 


\section{OPTIMAL CONTRACT MENU UNDER ASYMMETRIC COST INFORMATION}

Up until this point, we have analyzed the situations in which there is no IA. In this section, we drop the assumption of full information and analyze a more realistic case where the retailer possesses private information. We consider the setting where IA arises across one dimension: the supplier is uncertain about the retailer's cost type. There is a set of possible cost types that the retailer can have and this set is known to the supplier, i.e., the supplier is aware of the possible cost types, but is not aware of the retailer's actual cost type. He believes that the retailer can have $N$ types of cost with associated probabilities $P_{j}^{c} \geq 0$ for $j \in J=\{1,2, \ldots, N\}$ and $\sum_{j=1}^{N} P_{j}^{c}=1$. The retailer knows his cost type with certainty.

The situation where the supplier who lacks information moves first and offers a contract can be analyzed using a so-called "screening game" (see e.g., Chen (2003)). Instead of proposing a single contract as in the symmetric information case, the supplier offers a "menu of contracts", letting the retailer choose a specific contract from the menu, i.e., a "procurement plan-side payment" pair. In this way, the supplier persuades the retailer to truthfully reveal his type via the contract selected in the menu (self-selection).

Since the retailer has an incentive to misreport his cost parameters so as to receive a higher side payment, the optimal contracts the supplier offers under symmetric information are not "incentive compatible" under asymmetric information. Therefore, the supplier needs to ensure that the contract menu he offers does not lead to a misreport of cost parameters. To eliminate the possibility of cheating by the retailer, we rely on the "revelation principle" (Myerson 1981), stating that if there is an optimal contract for the supplier, then there exists an optimal contract which induces the retailer to truthfully reveal his cost type.

The sequence of events is as follows. At the outset, the retailer knows his cost type, unobserved by the supplier. The retailer and supplier observe customer demand. Knowing the retailer's possible cost types, the supplier can compute the retailer's outside option for each type $\left(\pi_{j}^{R *}\right)$. The supplier offers a menu with a contract $\left\{\left(x_{j, t}\right)_{t \in \mathcal{T}}, z_{j}\right\}$ for each assumed retailer type $j$ such that his expected profit is maximized. The retailer either selects one of the contracts in the menu, or imposes his own optimal plan on the supplier. The objective of the optimization problem is to maximize the supplier's expected profit.

\subsection{Formulation}

The supplier's problem of designing the optimal menu can be formulated as follows:

$$
\max \sum_{j=1}^{N}\left(P_{j}^{c}\left(\sum_{t=1}^{T}\left(p_{t}^{R} x_{j, t}^{R}-K_{t}^{S} y_{j, t}^{S}-p_{t}^{S} x_{j, t}^{S}-h_{t}^{S} I_{j, t}^{S}\right)-z_{j}\right)\right)
$$




$$
\begin{array}{rlrl}
\text { s.t. } \pi_{j \mid j}^{R}+z_{j} \geq \pi_{j}^{R *} & & \forall j \in J \\
\pi_{j \mid j}^{R}+z_{j} \geq \pi_{k \mid j}^{R}+z_{k} & \forall j, k \in J, j \neq k \\
\pi_{k \mid j}^{R}=\sum_{t=1}^{T}\left(s_{j, t}^{R} d_{t}-K_{j, t}^{R} y_{k, t}^{R}-p_{j, t}^{R} x_{k, t}^{R}-h_{j, t}^{R} I_{k, t}^{R}\right) & \forall j, k \in J \\
I_{j, t-1}^{R}+x_{j, t}^{R}=d_{t}+I_{j, t}^{R} & \forall t \in \mathcal{T}, \forall j \in J \\
x_{j, t}^{R} \leq d_{t, T} y_{j, t}^{R} & \forall t \in \mathcal{T}, \forall j \in J \\
x_{j, t}^{R} \geq y_{j, t}^{R} & \forall t \in \mathcal{T}, \forall j \in J \\
I_{j, t-1}^{S}+x_{j, t}^{S}=x_{j, t}^{R}+I_{j, t}^{S} & \forall t \in \mathcal{T}, \forall j \in J \\
x_{j, t}^{S} \leq d_{t, T} y_{j, t}^{S} & \forall t \in \mathcal{T}, \forall j \in J \\
x_{j, t}^{R} \in \mathbb{Z}_{+} & \forall t \in \mathcal{T}, \forall j \in J \\
z_{j}, x_{j, t}^{S}, I_{j, t}^{R}, I_{j, t}^{S}, \geq 0 & \forall t \in \mathcal{T}, \forall j \in J \\
y_{j, t}^{S}, y_{j, t}^{R} \in\{0,1\} & \forall t \in \mathcal{T}, \forall j \in J \\
I_{j, 0}^{R}, I_{j, 0}^{S}=0 & & \forall j \in J
\end{array}
$$

In this formulation, the decision variables have one additional index: $j \in J=\{1,2, \ldots, N\}$, representing the retailer's types, e.g., $x_{j, t}^{R}$ denotes the procurement quantity in the contract of retailer type $j$ in period $t$. The individual rationality constraint is formalized in (27). Each retailer's type has different cost parameters, and therefore it is reasonable to assume that the retailer's participation constraint is type-dependent. As mentioned earlier, our type-dependent individual rationality constraint is a major departure from much of the literature in which identical outside options for different retailer types are assumed (i.e., $\pi_{j}^{R *}=\pi^{R *}$ for all $j \in J$ ). The main reason to have such a "simple and debatable" (Laffont \& Martimort 2001) assumption is because of analytical tractability. However, because we use a mathematical programming solution approach, there is no reason to prefer identical outside options to the more realistic case of type-dependent ones.

Constraints (28) are the incentive compatibility (IC) constraints to ensure that the retailer will be worse off if he chooses a contract that is not designed for his own cost type.

The profit that the retailer realizes when he is of type $j$ and chooses the designed contract for type $k$ is defined in (29). The procurement quantities in the contracts offered are not necessarily integer, while we assume that the supply chain's final product is indivisible. Constraints (35) restrict the value of procurement quantities to be integers.

Constraints (32) ensure that the set-up variable is zero if there is no procurement in period $t$. It is noteworthy that in the outcomes of the model without considering this constraint, we have found some problem instances in which there is no procurement but there is a set-up, i.e., $x_{1, t}^{R}=0$ but $y_{1, t}^{R}=1$ (see Appendix for such an instance). The explanation is that although a cost is associated with the retailer's set-up variable, we do not directly optimize the retailer's total profit formalized by $\pi_{j \mid j}^{R}+z_{j}$. The following lemma shows that constraint (32) can be ignored in case all the retailer's types have the same fixed procurement cost across the whole planning 
horizon.

Lemma 5. If $K_{j, t}^{R}=K$ for all $t \in \mathcal{T}$ and $j \in J$, constraint (32) is redundant.

Proof: We prove the claim assuming two retailer types. It is easy to verify that the lemma holds in the case that there are more than two types. Suppose that the supplier offers a menu of two contracts, and in the contract designed for type 1 , we have a period $t \in \mathcal{T}$ in which $x_{1, t}^{R}=0$ and $y_{1, t}^{R}=1$ (there is no procurement but there is set-up). The relevant IR and IC constraints are:

$$
\begin{aligned}
& \pi_{\mid 11}^{R}+z_{1} \geq \pi_{1}^{R *} \\
& \pi_{2 \mid 2}^{R}+z_{2} \geq \pi_{2}^{R *} \\
& \pi_{1 \mid 1}^{R}+z_{1} \geq \pi_{2 \mid 1}^{R}+z_{2} \\
& \pi_{2 \mid 2}^{R}+z_{2} \geq \pi_{1 \mid 2}^{R}+z_{1} .
\end{aligned}
$$

Let $\pi_{j \mid k}^{R^{\prime}}$ and $z_{j}^{\prime}$ respectively denote for $j, k \in\{1,2\}$, the cost and side payment associated with the production plan in which $x_{1, t}^{R}=y_{1, t}^{R}=0$. We set $z_{1}^{\prime}=z_{1}-K_{1}^{R}$ and $z_{2}^{\prime}=z_{2}$. With $z_{1}^{\prime}$ and $z_{2}^{\prime}$, it is clear that:

$$
\pi_{1 \mid 1}^{R^{\prime}}=\pi_{1 \mid 1}^{R}+K_{1}^{R}, \quad \pi_{2 \mid 1}^{R^{\prime}}=\pi_{2 \mid 1}^{R}, \quad \pi_{1 \mid 2}^{R^{\prime}}=\pi_{1 \mid 2}^{R}+K_{2}^{R}, \quad \pi_{2 \mid 2}^{R^{\prime}}=\pi_{2 \mid 2}^{R} .
$$

With the assumption that $K_{j, t}^{R}=K$ for all $t \in \mathcal{T}$ and $j \in\{1,2\}$, profits in (43) and side payments associated with the new production plan satisfy constraints (39) to (42). We therefore have a new feasible solution with a larger profit.

It is noteworthy that $z_{1}^{\prime}=z_{1}-K_{1}^{R}$ is non-negative (satisfies constraint (36)), because $\pi_{1 \mid 1}^{R^{\prime}}$ is the profit associated with a feasible solution that cannot be larger than the retailer's maximum profit $\pi_{1}^{R *}$ and we also have

$$
z_{1} \geq \pi_{1}^{R *}-\pi_{1 \mid 1}^{R}=\pi_{1}^{R *}-\left(\pi_{1 \mid 1}^{R}+K_{1}^{R}\right)+K_{1}^{R}=\pi_{1}^{R *}-\pi_{1 \mid 1}^{R^{\prime}}+K_{1}^{R} \geq K_{1}^{R} .
$$

Similar to the model of the previous section, the feasible region of this problem is not empty. Consider the case where the supplier does not pay any side payment to the retailer, i.e., $z_{j}=0$ for all $j \in J$. This implies that constraints (27) would be binding. Additionally, the retailer will not be better off by pretending to be another cost type since there is no side payment. The solution of the above model is then the outcome of the uncoordinated SC, where the supplier has no option but to satisfy the retailer's individual optimal plan.

Example 6. Suppose that the retailer has 2 types of set-up cost shown in Table 3. The optimal ordering plans of the two types of the retailer and the supplier's production plan corresponding to each type with and without coordination are given in Table 4. With the assumption that each retailer cost type arises with the same probability, the expected profit of the supplier's outside option is $728.5(=1 / 2[791+666])$. The total expected profit of the supplier equals $926(=1 / 2[1418-349]+1 / 2[1399-616])$, leading to about $27 \%$ increase in his expected profit. Observe that the existence of IA does not allow the supplier to extract all the efficiency gains for himself. Rather, the supplier is obliged to give up some "information rent" to the retailer because of the latter's information advantage. In this example, there is information rent of $40(=1331+349-1640)$ units for 
the retailer's type 1 (the type with the higher set-up cost).

We also compute the expected profit improvement of the whole SC system. The SC profit if the retailer is actually of type 1 without coordination equals $2431(=1640+791)$, while the SC profit with coordination would be $2749(=1680+1069)$. In case the actual retailer cost is type 2 , the SC total profit in the case of sequential decision-making and the coordinated setting would be $3262(=2596+666)$ and $3379(=2596+783)$, respectively. Therefore, the expected SC profit under coordination is 3064, with about $8 \%$ profit improvement compared to the sequential decision-making setting. The expected SC total profit with a centralized decisionmaker is $3086(=1 / 2[2749+3423])$, and therefore the expected efficiency of the coordination mechanism is about 0.91 .

Table 3: Two cost types of the retailer

\begin{tabular}{cccccc}
\hline$t$ & 1 & 2 & 3 & 4 & 5 \\
\hline \hline$d_{t}$ & 26 & 25 & 29 & 33 & 77 \\
$p_{t}^{R}$ & 15 & 20 & 18 & 10 & 15 \\
$h_{t}^{R}$ & 10 & 16 & 10 & 15 & 13 \\
$s_{t}^{R}$ & 30 & 30 & 30 & 30 & 30 \\
\hline$K_{1, t}^{R}$ & 179 & 199 & 282 & 285 & 292 \\
$K_{2, t}^{R}$ & 29 & 85 & 29 & 43 & 21 \\
\hline
\end{tabular}

Table 4: Individual and coordinated optimal procurement plans (IA on cost)

\begin{tabular}{|c|c|c|c|c|c|c|c|c|c|}
\hline & & & & & & & \\
\hline & & & $x_{1}$ & $x_{2}$ & $x_{3}$ & $x_{4}$ & $x_{5}$ & $z$ & Profit \\
\hline \multirow{4}{*}{ 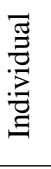 } & \multirow{4}{*}{$\frac{\tilde{E}}{2}$} & Retailer type 1 & 51 & 0 & 29 & 33 & 77 & - & 1640 \\
\hline & & Retailer type 2 & 26 & 25 & 29 & 33 & 77 & - & 2596 \\
\hline & & Supplier for retailer type 1 & 51 & 0 & 62 & 0 & 77 & - & 791 \\
\hline & & Supplier for retailer type 2 & 51 & 0 & 62 & 0 & 77 & - & 666 \\
\hline \multirow{4}{*}{ 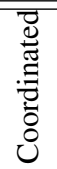 } & \multirow{4}{*}{$\frac{\text { जै }}{2}$} & Retailer type 1 contract & 51 & 0 & 62 & 0 & 77 & 349 & 1331 \\
\hline & & Retailer type 2 contract & 51 & 0 & 61 & 1 & 77 & 616 & 1980 \\
\hline & & Supplier for retailer type 1 & 51 & 0 & 62 & 0 & 77 & - & 1418 \\
\hline & & Supplier for retailer type 2 & 51 & 0 & 62 & 0 & 77 & - & 1399 \\
\hline
\end{tabular}

\subsection{Network interpretation}

In this subsection, we further analyze the optimization problem formulated in (26) - (38). In particular, the aim here is to examine which IC constraints are binding at the optimal solution and find a bound for the side payment quantities when the retailer possesses private information about one of his cost parameters. To do so, we rely on the rationalizability condition (Rochet 1987) and network interpretation (Vohra 2011) of the set of IC constraints. In order to demonstrate this interpretation, it is useful to rewrite the IC constraints in (28) as:

$$
z_{k}-z_{j} \leq \pi_{j \mid j}^{R}-\pi_{k \mid j}^{R} \quad \forall j, k \in J
$$

Together with the objective of minimizing the $z_{j}$ variables, these constraints coincide with the constraints of the dual of a longest path problem, where $z_{j}$ corresponds to the length of a longest path. However, since it is more common to work with a shortest path problem, in our analysis, we consider $-z_{j}$ which corresponds to the length of a shortest path. In view of this observation, we introduce a vertex $j$ for each type $j \in J$. Between every ordered pair of vertices $(k, j)$, we insert a directed arc of length $\ell(k, j)=\pi_{j \mid j}^{R}-\pi_{k \mid j}^{R}$. The IR constraints can be incorporated by assuming a dummy type of value 0 with $z_{0}=0$. Figure 1 shows a part of this network. 
For a fixed set of procurement plans $\left(\boldsymbol{x}_{\boldsymbol{j}}^{\boldsymbol{R}}\right)_{j \in J}$, the set of IC constraints is feasible iff this network has no negative-length cycle. If the network does have a negative cycle, there is no set of side payments that makes the procurement plans incentive compatible. In a network without negative-length cycles, each $-z_{j}$ is upper bounded by the length of the shortest path from the source vertex to vertex $j$. Therefore the optimal choice of $z_{j}$ would be to set it equal to minus the the length of the shortest path. To ensure that IR constraints hold, we choose the source to be the vertex corresponding to the dummy type 0. Arcs on the shortest path tree (union of all shortest paths from the source to all vertices) correspond to binding IC constraints.

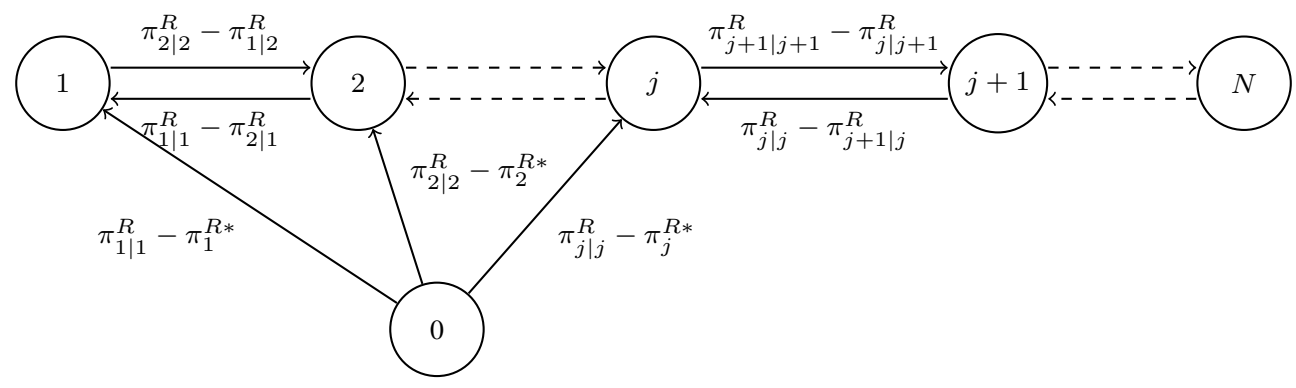

Figure 1: Network of types

Next, we discuss the relevant IC constraints and identify the ones that are redundant. Before that, we present two definitions for a set of ordered retailer types with time-invariant private cost information.

Definition 7. Monotonic procurement plans. The definition of monotonic procurement plans varies with the retailer's private information:

(a) Suppose the retailer has private information about his holding cost. For ordered holding costs, a contract menu consists of monotonic procurement plans if the corresponding total ending inventories across the whole planning horizon are monotonic. That is, if $h_{j}^{R}<h_{j+1}^{R}$ then $\sum_{t=1}^{T} I_{j, t}^{R} \geq \sum_{t=1}^{T} I_{j+1, t}^{R}$.

(b) Suppose the retailer has private information about his set-up cost. For ordered set-up costs, a contract menu consists of monotonic procurement plans if the corresponding total numbers of set-ups across the whole planning horizon are monotonic. That is, if $K_{j}^{R}<K_{j+1}^{R}$ then $\sum_{t=1}^{T} y_{j, t}^{R} \geq \sum_{t=1}^{T} y_{j+1, t}^{R}$.

Definition 8. Local IC constraints. With ordered types, e.g., $h_{j-1}^{R}<h_{j}^{R}<h_{j+1}^{R}$, IC constraints can be divided into two sets: downward IC (DIC) constraints in which the type $j$ retailer chooses the contract designed for a type with a lower cost and upward IC (UIC) constraints in which type $j$ retailer chooses the contract designed for a type with a higher cost. Local DIC and UIC constraints are:

$$
\begin{array}{lll}
\pi_{j \mid j}^{R}+z_{j} \geq & \pi_{j-1 \mid j}^{R}+z_{j-1} & \forall j \in\{2, \ldots, N\} \\
\pi_{j \mid j}^{R}+z_{j} \geq & \pi_{j+1 \mid j}^{R}+z_{j+1} & \forall j \in\{1,2, \ldots, N-1\} .
\end{array}
$$

As mentioned earlier, given a set of procurement plans, we have a way to check whether the plans are incentive compatible: we need to ensure that the network corresponding to the IC constraints does not contain negative-length cycles. Theorem 9 shows the necessary and sufficient condition under which there is no negative- 
length cycle between a pair of vertices. Consequently, Theorem 11 shows that with ordered types, the global IC constraints are implied by the local ones.

Theorem 9. A network of types associated with the system (44) does not contain negative-length cycles between pairs of vertices iff the procurements plans in a menu of contracts are monotonic.

Proof: We prove the claim for the case of IA on the retailer's holding costs. A similar argument can be used to prove the claim for other private cost parameters. Consider the non-negative cycle condition applied to cycle $j \rightarrow j+1 \rightarrow j$ in Figure 1. With the assumption of time-invariant holding cost, it implies:

$$
\begin{array}{r}
\ell(j, j+1)+\ell(j+1, j) \geq 0 \Leftrightarrow \pi_{j+1 \mid j+1}^{R}-\pi_{j \mid j+1}^{R}+\pi_{j \mid j}^{R}-\pi_{j+1 \mid j}^{R} \geq 0 \\
\Leftrightarrow \sum_{t=1}^{T}\left(s_{t}^{R} d_{t}-K_{t}^{R} y_{j+1, t}^{R}-p_{t}^{R} x_{j+1, t}^{R}-h_{j+1}^{R} I_{j+1, t}^{R}\right)-\sum_{t=1}^{T}\left(s_{t}^{R} d_{t}-K_{t}^{R} y_{j, t}^{R}-p_{t}^{R} x_{j, t}^{R}-h_{j+1}^{R} I_{j, t}^{R}\right)+ \\
\sum_{t=1}^{T}\left(s_{t}^{R} d_{t}-K_{t}^{R} y_{j, t}^{R}-p_{t}^{R} x_{j, t}^{R}-h_{j}^{R} I_{j, t}^{R}\right)-\sum_{t=1}^{T}\left(s_{t}^{R} d_{t}-K_{t}^{R} y_{j+1, t}^{R}-p_{t}^{R} x_{j+1, t}^{R}-h_{j}^{R} I_{j+1, t}^{R}\right) \geq 0 \\
\Leftrightarrow \sum_{t=1}^{T}\left(h_{j+1}^{R}-h_{j}^{R}\right) I_{j, t}^{R}-\sum_{t=1}^{T}\left(h_{j+1}^{R}-h_{j}^{R}\right) I_{j+1, t}^{R} \geq 0 \\
\Leftrightarrow\left(h_{j+1}^{R}-h_{j}^{R}\right)\left(\sum_{t=1}^{T} I_{j, t}^{R}-\sum_{t=1}^{T} I_{j+1}^{R}\right) \geq 0 \Leftrightarrow \sum_{t=1}^{T} I_{j, t}^{R} \geq \sum_{t=1}^{T} I_{j+1, t}^{R} .
\end{array}
$$

The proof for a cycle on a pair of non-consecutive vertices, i.e., $j \rightarrow j+k \rightarrow j, k>1$ is similar.

Next, we show that the retailer's revenue has a specific property which will be used to simplify the set of IC constraints.

Lemma 10. The retailer's revenue $\pi_{j \mid k}^{R}$ for types $j, k \in J$ satisfies the increasing differences property.

Proof: The claim that we need to prove varies with the retailer's private information. We need to show that given two ordered types of retailer and two monotonic procurement plans, $x_{j}^{R}$ and $x_{k}^{R}$, we have

$$
\pi_{k \mid k^{\prime}}^{R}-\pi_{j \mid k^{\prime}}^{R} \geq \pi_{k \mid j^{\prime}}^{R}-\pi_{j \mid j^{\prime}}^{R}
$$

where

(a) the retailer has private information about his holding cost and $h_{j^{\prime}}^{R}<h_{k^{\prime}}^{R}$,

(b) the retailer has private information about his set-up cost and $K_{j^{\prime}}^{R}<K_{k^{\prime}}^{R}$.

We prove the claim in part (a). The proof of part (b) is similar. After substituting in the expression for $\pi_{j \mid k}^{R}$ and canceling common terms, the inequality (45) is equivalent to:

$$
\left(h_{k^{\prime}}^{R}-h_{j^{\prime}}^{R}\right)\left(\sum_{t=1}^{T} I_{j, t}^{R}-\sum_{t=1}^{T} I_{k, t}^{R}\right) \geq 0,
$$

which holds by the monotonicity of total ending inventory quantities and the fact that $h_{j^{\prime}}^{R}<h_{k^{\prime}}^{R}$.

Theorem 11. Suppose that procurement plans are monotonic and therefore $\pi_{j \mid k}^{R}$ satisfies increasing differences. Only the local IC constraints matter out of all the IC constraints.

Proof: We prove the claim for the case of IA on holding cost. A similar argument can be used to prove the claim for private set-up cost. With ordered types, i.e., $h_{j-1}^{R} \leq h_{j}^{R}$, we show that only the local DIC constraints 
are sufficient. To do so, we show that the following pair of constraints:

$$
\begin{aligned}
\pi_{j \mid j}^{R}+z_{j} & \geq \pi_{j-1 \mid j}^{R}+z_{j-1} \\
\pi_{j-1 \mid j-1}^{R}+z_{j-1} & \geq \pi_{j-2 \mid j-1}^{R}+z_{j-2}
\end{aligned}
$$

imply that $\pi_{j \mid j}^{R}+z_{j} \geq \pi_{j-2 \mid j}^{R}+z_{j-2}$. The rest of DIC constraints will follow by induction and non-negativity of cycles in the network. Adding (46) and (47) and rearranging yields

$$
\pi_{j \mid j}^{R}+z_{j} \geq\left(\pi_{j-1 \mid j}^{R}-\pi_{j-2 \mid j}^{R}\right)-\left(\pi_{j-1 \mid j-1}^{R}-\pi_{j-2 \mid j-1}^{R}\right)+\pi_{j-2 \mid j}^{R}+z_{j-2} .
$$

The increasing differences property and monotonicity of total ending inventories imply that

$$
\left(\pi_{j-1 \mid j}^{R}-\pi_{j-2 \mid j}^{R}\right)-\left(\pi_{j-1 \mid j-1}^{R}-\pi_{j-2 \mid j-1}^{R}\right) \geq 0,
$$

and therefore we have

$$
\pi_{j \mid j}^{R}+z_{j} \geq \pi_{j-2 \mid j}^{R}+z_{j-2}
$$

Using similar arguments, we can show that global UIC constraints are implied by the local ones.

Theorems 9 and 11 imply that we can replace the set of IC constraints with the corresponding monotonicity requirement of procurement plans (see Definition 7) together with the set of local IC constraints.

To summarize, when a set of procurement plans is incentive compatible (there is no negative-length cycle in the network of types), minus the length of the shortest path to a vertex (type) is the side payment quantity that the supplier pays to the retailer while preserving incentive compatibility. To find all the shortest paths from vertex 0 to each vertex $j \in J$, instead of taking into account the full set of IC constraints, we have shown that it is sufficient to only consider the subset of local IC constraints. This means that it is sufficient to ensure that each retailer type has no incentive to make a "small" misreport of his type. This property enables us to considerably reduce the number of IC constraints from a quadratic number of $n(n-1)$ constraints to a more tractable set with a linear number of $2(n-1)$ constraints.

Example 12. Suppose the retailer has private information about his time-invariant holding cost. The supplier assumes 4 possible cost parameters for the retailer, arising with the same probability: $h_{1}^{R}=1, h_{2}^{R}=5, h_{3}^{R}=10$, and $h_{4}^{R}=32$. Table 5 shows demand and other cost parameters of the retailer. The supplier's cost parameters are given in Table $2 \mathrm{~b}$. The network corresponding to this example is depicted in Figure 2. The dashed arcs form the shortest path tree and show the binding IC and IR constraints. Considering the length of the shortest path from vertex 0 to each vertex, we have $z_{1} \geq 117, z_{2} \geq 299, z_{3} \geq 79$ and $z_{4} \geq 0$. Table 6 shows the optimal menu of contracts.

Table 5: Parameters of the retailer's problem in Example 12

\begin{tabular}{cccccc}
\hline$t$ & 1 & 2 & 3 & 4 & 5 \\
\hline \hline$d_{t}$ & 26 & 25 & 29 & 33 & 11 \\
$K_{t}^{R}$ & 150 & 89 & 182 & 165 & 198 \\
$p_{t}^{R}$ & 5 & 6 & 7 & 5 & 9 \\
$s_{t}^{R}$ & 15 & 20 & 22 & 21 & 19 \\
\hline
\end{tabular}




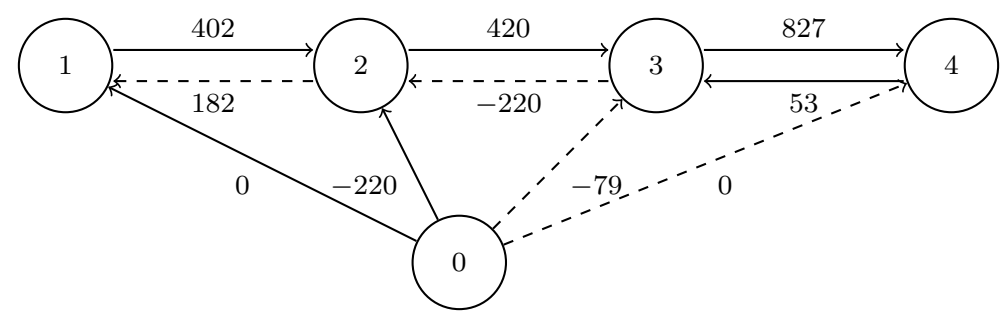

Figure 2: Network of types in Example 12

Table 6: Optimal menu of contracts in Example 12

\begin{tabular}{lcccccccc}
\hline Contract & $x_{1}$ & $x_{2}$ & $x_{3}$ & $x_{4}$ & $x_{5}$ & $z$ & $\pi_{j, j}^{R}$ & $\pi_{j}^{R *}$ \\
\hline \hline Retailer type 1 & 124 & 0 & 0 & 0 & 0 & 117 & 1434 & 1434 \\
Retailer type 2 & 51 & 0 & 73 & 0 & 0 & 299 & 932 & 1152 \\
Retailer type 3 & 26 & 54 & 0 & 44 & 0 & 79 & 952 & 1031 \\
Retailer type 4 & 26 & 25 & 29 & 33 & 11 & 0 & 899 & 899 \\
\hline
\end{tabular}

Note that with the simplifying assumption that the outside options of different retailer types are identical, i.e., $\pi_{j}^{R *}=\pi^{R *}$ for $j \in J$, there is only one outgoing arc from vertex 0 which goes to the vertex corresponding to the most inefficient (the one with the highest cost) type. Because if a menu of contracts allows an inefficient type to reach his outside option, it will be automatically the case for the efficient types that have lower costs. Then the shortest path tree rooted at vertex 0 must be a unique path of $0 \rightarrow N \rightarrow \ldots \rightarrow 2 \rightarrow 1$. This implies that $z_{N}=$ $-\left(\pi_{N \mid N}^{R}-\pi_{N}^{R *}\right)$ and for each type $j<N$, the side payment is equal to $z_{j}=-\sum_{j \leq r \leq N-1}\left(\pi_{r \mid r}^{R}-\pi_{r+1 \mid r}^{R}\right)+z_{N}$. In this case, the problem is considerably simplified as the $z_{j}$ variables can be eliminated from the formulation by rewriting them in terms of the retailer's profit. As shown in Figure 2, in the absence of a common outside option, any of the arcs rooted at vertex 0 can be part of the shortest path tree.

The next step that we take is to examine whether the set of IC constraints can be further reduced. Consider Example 12 and observe that the horizontal dashed arcs in Figure 2 correspond to the local upward IC (or LUIC) constraints in which the efficient type pretends to be inefficient. This observation is commonly used in the mechanism design literature to further reduce the set of IC constraints and uncover some (qualitative) properties of optimal contracts. Although we can show in our setting that the total profit of the designed contract for an efficient type is not smaller than the profit of an inefficient type, i.e., $\pi_{j \mid j}^{R}+z_{j} \geq \pi_{j^{\prime} \mid j^{\prime}}^{R}+z_{j^{\prime}}$ for $j, j^{\prime} \in J, j<j^{\prime}$, this does not allow us to conclude that the efficient type's information rent $\left(\pi_{j^{\prime} \mid j^{\prime}}^{R}+z_{j^{\prime}}-\pi_{j^{\prime}}^{R *}\right)$ is in general (weakly) larger than the information rent of the inefficient type. Therefore, we cannot exclude the possibility that the inefficient type pretends to be efficient. This unexpected result can be attributed to the consideration of the dependence between the retailer's private information and his outside option. The next example shows an instance in which the inefficient type pretends to be efficient. The consequence of this observation is that no further simplification of the set of IC constraints is possible in our setting.

Example 13. Suppose the retailer has private information about his time-invariant holding cost. The supplier assumes 2 possible cost parameters with the same probability: $h_{1}^{R}=4.9, h_{2}^{R}=7.6$. We have $h_{t}^{S}=h^{S}=1$, $p_{t}^{R}=p_{t}^{S}=p=0$, and $s_{t}^{R}=s^{R}=5$. Table 7 shows demand and set-up cost of the retailer and supplier. The 
associated network of types is illustrated in Figure 3. The dashed arc from vertex 1 to 2 represents the binding IC constraint in which type 2 (inefficient type) chooses the contract designed for type 1 (efficient type). Table 8 shows the optimal menu. Observe that the inefficient type's information rent is 1 unit while there is no rent for the efficient type.

Table 7: Parameters of the problem in Example 13

\begin{tabular}{cccccc}
\hline$t$ & 1 & 2 & 3 & 4 & 5 \\
\hline \hline$d_{t}$ & 5 & 6 & 12 & 6 & 20 \\
$K_{t}^{S}$ & 151 & 40 & 186 & 50 & 190 \\
$K_{t}^{R}$ & 38 & 38 & 38 & 38 & 38 \\
\hline
\end{tabular}

Table 8: Optimal menu of contracts in Example 13

\begin{tabular}{lcccccccc}
\hline Contract & $x_{1}$ & $x_{2}$ & $x_{3}$ & $x_{4}$ & $x_{5}$ & $z$ & $\pi_{j, j}^{R}$ & $\pi_{j}^{R *}$ \\
\hline \hline Retailer type 1 & 11 & 0 & 12 & 6 & 20 & 8.6 & 63.6 & 72.2 \\
Retailer type 2 & 5 & 6 & 12 & 6 & 20 & 1 & 55 & 55 \\
\hline & & & & & & & &
\end{tabular}

Figure 3: Network of types in Example 13

\section{OPTIMAL CONTRACT MENU WITH TWO DIMENSIONS OF INFORMATION ASYMMETRY}

Rather than only having one-dimensional uncertainty about a cost parameter, in this section we analyze the case that the supplier is uncertain about an additional dimension: another cost parameter or customer demand at the retailer's level. While most of the studies with IA in the operations management literature analyze the problems with private information about either cost parameters or demand, in this section, we consider both cost and demand uncertainty. To the best of our knowledge, the study by Kostamis \& Duenyas (2011) is the only exception in the literature with IA about both a cost parameter and market demand. Although the two dimensions of IA in this study is similar to ours, our analysis differs in two important ways. First, we have a multi-period model with a discrete, rather than a binary, type space for each dimension. Second, we do not assume a common outside option for all the retailer types.

In subsection 7.1, we formulate the supplier's problem of designing optimal contract menus with two dimensions of IA. Next, subsection 7.2 presents the network interpretation.

\subsection{Formulation}

As in the previous section, suppose that the supplier is uncertain about a cost parameter. He believes that the retailer can have $N$ cost types with the associated probabilities $P_{j}^{c} \geq 0$ for $j \in J=\{1,2, \ldots, N\}$ and $\sum_{j=1}^{N} P_{j}^{c}=1$. In 7.1.1, we present the formulation of the two-dimensional IA setting where customer demand is also uncertain. This is followed by the discussion of the model with private set-up and holding cost information 
in 7.1.2. The type of the retailer throughout this section is identified by a pair of indices, each representing a dimension of private information.

\subsubsection{Asymmetric cost and demand information:}

The supplier believes that the retailer can have $M$ demand scenarios with the associated probabilities $P_{i}^{d} \geq 0$ for $i \in I=\{1,2, \ldots, M\}$ and $\sum_{i=1}^{M} P_{i}^{d}=1$. The retailer knows his demand scenario with certainty. With the assumption that demand and cost types are independent, the supplier's beliefs satisfy $P_{i, j}=\left(P_{i}^{d}\right)\left(P_{j}^{c}\right)$ for $i \in I$, $j \in J$ and $\sum_{i \in I, j \in J} P_{i, j}=1$.

Incorporation of demand uncertainty in the model requires us to keep track of shortages that may occur at the retailer's level. We consider the case where there are stockouts, i.e., demand is not satisfied or backlogged but lost. We introduce a new decision variable and a new parameter. $L_{i^{\prime}, j^{\prime}, t}^{R, i, j}$ is the quantity of unsatisfied demand in period $t$ of retailer type $(i, j)$, who chooses the contract designed for the retailer of type $\left(i^{\prime}, j^{\prime}\right)$. Parameter $s_{j, t}^{R}$ accounts for the stockout cost of the retailer of type $j$ in period $t$. Stockout costs are not easy to quantify and typically measured by the selling price (see e.g., Aksen et al. (2003)).

In this model, the retailer's inventory level depends not only on the actual cost type and demand scenario, but also on the chosen contract: $I_{i^{\prime}, j^{\prime}, t}^{R, i, j}$ denotes the ending inventory level in period $t$ of retailer type $(i, j)$ who chooses the contract designed for the retailer type $\left(i^{\prime}, j^{\prime}\right)$.

The retailer's revenue in a period $t$ is the product of the realized sales and the corresponding unit selling price. The realized sale in period $t$ is given by $d_{i, t}-L_{i^{\prime}, j^{\prime}, t}^{R, i, j}$, demand minus shortage in period $t$. With the assumption that $s_{j, t}^{R}$ denotes the unit revenue in period $t$, the retailer's revenue is $\sum_{t \in \mathcal{T}} s_{j, t}^{R} d_{i, t}$.

The sequence of events is as follows. At the beginning, the retailer knows his cost type, unobserved by the supplier. The retailer observes customer demand. Being aware of the possible cost and demand types, the supplier computes the optimal ordering profit of the retailer for each type $\left(\pi_{i, j}^{R *}\right)$. Then he offers a menu of "procurement plan-side payment" contracts $\left\{\left(x_{i, j, t}\right)_{t \in \mathcal{T}}, z_{i, j}\right\}$ for each pair of assumed retailer type $(i, j)$ such that his expected profit is maximized. The retailer either selects one of the contracts in the menu or imposes his own optimal plan on the supplier.

As the supplier moves first and offers a menu of contracts, he needs to consider the retailer's reaction to the proposed offer in his optimization problem. Therefore, in the current setting with two dimensions of private information, the supplier's optimization problem entails a nested (bi-level) optimization task that corresponds to the retailer's optimization problem. The upper level optimization task corresponds to the supplier's problem, and the lower level optimization task corresponds to the retailer's problem.

In the model with one dimension of private cost information, we do not need to consider a bi-level optimization problem since by choosing a contract, the retailer's optimal procurement plan, and hence the corresponding inventory levels are fully specified. However, this is not the case in the setting with demand uncertainty. Given both the procurement quantities in the chosen contract (which is not targeted to his real type) and the real demand scenarios, the retailer needs to determine how much demand to lose, when to lose this demand, and how much 
inventory to hold in each period such that his total profit is maximized. That is, the optimal quantities of $y_{i, j, t}^{R}$, $L_{i^{\prime}, j^{\prime}, t}^{R, i, j}$, and $I_{i^{\prime}, j^{\prime}, t}^{R, i, j}$ need to be specified.

The supplier's bi-level optimization problem can be formulated as:

$$
\begin{array}{rlrl}
\max \sum_{i=1}^{M} \sum_{j=1}^{N} P_{i, j}\left(\sum_{t=1}^{T}\left(p_{t}^{R} x_{i, j, t}^{R}-K_{t}^{S} y_{i, j, t}^{S}-p_{t}^{S} x_{i, j, t}^{S}-h_{t}^{S} I_{i, j, t}^{S}\right)-z_{i, j}\right) & \\
\text { s.t. } & \pi_{i, j}^{R, i, j}+z_{i, j} \geq \pi_{i, j}^{R *} & \forall i \in I, \forall j \in J \\
\pi_{i, j}^{R, i, j}+z_{i, j} \geq \pi_{i^{\prime}, j^{\prime}}^{R, i}+z_{i^{\prime}, j^{\prime}} & \forall i, i^{\prime} \in I, \forall j, j^{\prime} \in J \\
I_{i, j, t-1}^{S}+x_{i, j, t}^{S}=x_{i, j, t}^{R}+I_{i, j, t}^{S} & \forall t \in \mathcal{T}, \forall i \in I, \forall j \in J \\
x_{i, j, t}^{S} \leq \max _{k \in I}\left\{d_{k, 1, T}\right\} y_{i, j, t}^{S} & \forall t \in \mathcal{T}, \forall i \in I, \forall j \in J \\
x_{i, j, t}^{R} \in \mathbb{Z}_{+} & \forall t \in \mathcal{T}, \forall i \in I, \forall j \in J \\
z_{i, j}, x_{i, j, t}^{S}, I_{i, j, t}^{S} \geq 0 & \forall t \in \mathcal{T}, \forall i \in I, \forall j \in J \\
y_{i, j, t}^{S} \in\{0,1\} & \forall t \in \mathcal{T}, \forall i \in I, \forall j \in J \\
I_{i, j, 0}^{S}=0 & \forall i \in I, \forall j \in J
\end{array}
$$

in which $\pi_{i, j}^{R, i, j}$ is the outcome of the following optimization problem:

$$
\begin{aligned}
\pi_{i^{\prime}, j^{\prime}}^{R, i, j}=\max \sum_{t=1}^{T}\left(s_{j, t}^{R} d_{i, t}-K_{j, t}^{R} y_{i^{\prime}, j^{\prime}, t}^{R}-p_{t}^{R} x_{i^{\prime}, j^{\prime}, t}^{R}-h_{j, t}^{R} I_{i^{\prime}, j^{\prime}, t}^{R, i, j}-s_{j, t}^{R} L_{i^{\prime}, j^{\prime}, t}^{R, i j}\right) & \forall i, i^{\prime} \in I, \forall j, j^{\prime} \in J \\
\text { s.t. } \quad & \forall t \in \mathcal{T}, \forall i, i^{\prime} \in I, \forall j, j^{\prime} \in J \\
I_{i^{\prime}, j^{\prime}, t-1}^{R, i, j}+x_{i^{\prime}, j^{\prime}, t}^{R}=d_{i, t}+I_{i^{\prime}, j^{\prime}, t}^{R, i, j}-L_{i^{\prime}, j^{\prime}, t}^{R, i, j} & \forall t \in \mathcal{T}, \forall i \in I, \forall j \in J \\
x_{i, j, t}^{R} \leq \max _{k \in I}\left\{d_{k, 1, T}\right\} y_{i, j, t}^{R} & \forall t \in \mathcal{T}, \forall i, i^{\prime} \in I, \forall j, j^{\prime} \in J \\
L_{i^{\prime}, j^{\prime}, t}^{R, i, j} \leq d_{i, t} & \forall t \in \mathcal{T}, \forall i, i^{\prime} \in I, \forall j, j^{\prime} \in J \\
I_{i^{\prime}, j^{\prime}, t}^{R, i, j}, L_{i^{\prime}, j^{\prime}, t} \geq 0 & \forall t \in \mathcal{T}, \forall i \in I, \forall j \in J \\
y_{i, j, t}^{R} \in\{0,1\} & \forall i, i^{\prime} \in I, \forall j, j^{\prime} \in J \\
I_{i^{\prime}, j^{\prime}, 0}^{R, i, j}=0 &
\end{aligned}
$$

The decision variables in this bi-level problem have one more index $i \in I=\{1,2, \ldots, M\}$, representing demand scenarios. For instance, $x_{i, j, t}^{R}$ is the procurement quantity in the contract of the retailer type $(i, j)$ in period $t . \pi_{i^{\prime}, j^{\prime}}^{R, i, j}$ (in (57)) is the profit of the retailer of type $(i, j)$ who chooses the contract for the retailer type $\left(i^{\prime}, j^{\prime}\right)$. In the set-up forcing constraints (52) and (59), the maximum total demand across all the possible demand scenarios is considered as an upper bound for the procurement quantities in each period $t$. Constraints (60) ensure that the retailer's stockout quantity in period $t$ does not exceed the demand of that period.

To transform the bi-level optimization problem into a single-level problem, we consider the lower level (retailer's) problem in more detail. For clarity, we leave out all the subscripts and superscripts representing the retailer types. The retailer's optimization problem is as follows:

$$
\max _{y_{t}, I_{t}, L_{t}} \sum_{t=1}^{T}\left(s_{t} d_{t}-K_{t} y_{t}-p_{t} x_{t}-h_{t} I_{t}-s_{t} L_{t}\right)
$$




\begin{tabular}{|c|c|c|}
\hline s.t. & $I_{t-1}+x_{t}+L_{t}=d_{t}+I_{t}$ & $\forall t \in \mathcal{T}$ \\
\hline & $x_{t} \leq y_{t} d_{1, T}$ & $\forall t \in \mathcal{T}$ \\
\hline & $L_{t} \leq d_{t}$ & $\forall t \in \mathcal{T}$ \\
\hline & $I_{t}, L_{t} \geq 0$ & $\forall t \in \mathcal{T}$ \\
\hline & $y_{t} \in\{0,1\}$ & $\forall t \in \mathcal{T}$ \\
\hline & $I_{0}=0$ & \\
\hline
\end{tabular}

In this formulation, $x_{t}$ is given in the contract. The retailer also knows his real demand scenario and he solves the above integer linear program to find the optimal quantities of $y_{t}, L_{t}$, and $I_{t}$.

Next we demonstrate that the retailer's optimization problem can be written as a system of linear (in)equalities. The following theorems show that there is one unique optimal solution, satisfying the property that $L_{t} I_{t}=0$ for $t \in \mathcal{T}$. First, Theorem 14 shows that we have an optimal solution such that there is no ending inventory in a given period if demand is not fully satisfied in that period. In order for this property to hold, we need a mild assumption on the retailer's cost parameters.

Assumption 1. There is no speculative motive for the retailer to postpone satisfying demand, i.e., the stockout cost of one unit of demand in some period plus the cost of holding one unit inventory until some future period is at least the stockout cost in the latter period: $\sum_{j=t}^{v-1} h_{j}+s_{t} \geq s_{v}$.

Theorem 14. Given Assumption 1, there exists an optimal solution such that $L_{t} I_{t}=0$ for $t \in \mathcal{T}$.

Proof: By substituting the inventory balance constraint $I_{t-1}+x_{t}+L_{t}=d_{t}+I_{t}$ into the objective function in (64), we have

$$
\begin{aligned}
& \sum_{t=1}^{T}\left(s_{t} d_{t}-K_{t} y_{t}-p_{t} x_{t}-h_{t} I_{t}-s_{t}\left(d_{t}+I_{t}-I_{t-1}-x_{t}\right)\right) \\
= & \sum_{t=1}^{T-1}-\left(h_{t}+s_{t}-s_{t+1}\right) I_{t}-\left(h_{T}+s_{T}\right) I_{T}-\sum_{t=1}^{T}\left(K_{t} y_{t}+\left(p_{t}-s_{t}\right) x_{t}\right)
\end{aligned}
$$

The aim is to maximize this function and since $h_{t}+s_{t}-s_{t+1} \geq 0$ under assumption 1 , we need to have the optimal value of $I_{t}$ as small as possible and non-negative. This implies that we should satisfy as much of demand as we can and therefore we will have

$$
\begin{cases}L_{t}^{*}=0 \text { and } I_{t}^{*}=I_{t-1}^{*}+x_{t}-d_{t} & \text { if } I_{t-1}^{*}+x_{t}-d_{t}>0 \\ I_{t}^{*}=0 \text { and } L_{t}^{*}=d_{t}-I_{t-1}^{*}-x_{t} & \text { if } I_{t-1}^{*}+x_{t}-d_{t} \leq 0\end{cases}
$$

This implies that we have an optimal solution with the desired property.

We proved that the optimal solution has a specific property, the following theorem shows that there is indeed one solution that satisfies the property.

Theorem 15. There exists only one feasible solution that satisfies $L_{t} I_{t}=0$ for $t \in \mathcal{T}$.

Proof: Any feasible solution should satisfy the inventory balance constraint 


$$
I_{t-1}+x_{t}+L_{t}=d_{t}+I_{t} \quad \forall t \in \mathcal{T}
$$

Consider period 1 in which $I_{0}=0$ and thus the inventory balance constraint would be $L_{1}=I_{1}+d_{1}-x_{1}$. The quantity of $x_{1}$ is specified in the contract and the retailer knows his demand $d_{1}$. The quantity of $L_{1}$ can be uniquely specified using (65). There exists only one feasible solution in period 1 that has the desired property. Now $I_{1}$ is specified, $x_{2}$ and $d_{2}$ are known, and what remains is to determine the value of $L_{2}$ and $I_{2}$ in a similar way. Following the same reasoning for the subsequent periods, we can show that in each period $t \in \mathcal{T}$, there exists only one feasible solution that satisfies $L_{t} I_{t}=0$.

To ensure that there is no optimal solution that does not satisfy Theorem 14, we assume that by holding inventory, the retailer certainly will incur holding cost, i.e., $h_{t}>0$ for $t \in \mathcal{T}$.

The above theorems can be used to rewrite the retailer's problem as a system of linear (in)equalities. We can enforce that the property of the unique optimal solution of the retailer's problem $\left(L_{t} I_{t}=0, \forall t \in \mathcal{T}\right)$ holds in the optimization problem of the supplier. To do so, we introduce two new binary variables:

$$
\begin{aligned}
& u_{i^{\prime}, j^{\prime}, t}^{i, j}: 1 \text { if } L_{i^{\prime}, j^{\prime}, t}^{R, i, j}>0 \text { and } 0 \text { otherwise } \\
& v_{i^{\prime}, j^{\prime}, t}^{i, j}: 1 \text { if } I_{i^{\prime}, j^{\prime}, t}^{R, i, j}>0 \text { and } 0 \text { otherwise. }
\end{aligned}
$$

To have the desired property, we also consider new constraints as follows:

$$
\begin{array}{rlrl}
L_{i^{\prime}, j^{\prime}, t}^{R, i, j} & \leq \max _{k \in I}\left\{d_{k, 1, T}\right\} u_{i^{\prime}, j^{\prime}, t}^{i, j} & & \forall t \in \mathcal{T}, \forall i, i^{\prime} \in I, \forall j, j^{\prime} \in J \\
I_{i^{\prime}, j^{\prime}, t}^{R, i, j} & \leq \max _{k \in I}\left\{d_{k, 1, T}\right\} v_{i^{\prime}, j^{\prime}, t}^{i, j} & & \forall t \in \mathcal{T}, \forall i, i^{\prime} \in I, \forall j, j^{\prime} \in J \\
u_{i^{\prime}, j^{\prime}, t}^{i, j}+v_{i^{\prime}, j^{\prime}, t}^{i, j} & \leq 1 & & \forall t \in \mathcal{T}, \forall i, i^{\prime} \in I, \forall j, j^{\prime} \in J \\
u_{i^{\prime}, j^{\prime}, t}^{i, j}, v_{i^{\prime}, j^{\prime}, t}^{i, j} & \in\{0,1\} & \forall t \in \mathcal{T}, \forall i, i^{\prime} \in I, \forall j, j^{\prime} \in J
\end{array}
$$

Instead of a bi-level optimization problem, the supplier solves the single-level MIP, consisting of (48) - (63) plus (66) - (69), in order to find his optimal menu of contracts.

Two remarks are in order regarding this single-level MIP model. (1) Similar to the one-dimensional model, in order to have a correct formulation, we need to consider the set of constraints $x_{i, j, t}^{R} \geq y_{i, j, t}^{R}, \forall t \in \mathcal{T}, \forall i \in$ $I, \forall j \in J$ in the supplier's problem. These constraints are redundant if all the retailer's types have the same fixed procurement cost across the whole planning horizon, i.e., $K_{j, t}^{R}=K$ for $t \in \mathcal{T}$ and $j \in J$. (2) Following the reasoning in Section 6, the feasible region of this problem is not empty.

Example 16. Suppose that there are 2 demand scenarios with equal probability of 0.5 according to Table 9 . The retailer's time-invariant holding cost of type 1 and type 2 are 3.4 and 8.7 units, respectively. Other cost parameters are given in Table 10. Table 11 shows the individual and coordinated optimal procurement plans. The expected supplier's outside option equals $329.5(=1 / 4[234.4-3.4+485.5+601.5])$. The supplier's expected profit with coordination equals $932.2(=1 / 4[[641.2-157.3]+[681.8-179.7]+[1598.8-257.4]+[1588.8-187.4]])$, leading to approximately 3 times larger profit for the supplier. The expected SC total profit with respect to the 4 possible 
combinations of cost type and demand scenario when there is no coordination is 601 , while the expected profit with coordination equals 1203.7 . In the ideal case with a centralized decision-maker, the expected SC total profit equals 1213.5 and therefore the expected coordination mechanism efficiency is about 0.98 .

To investigate the effect of one and two-dimensional IA on the retailer's profit, consider the retailer type $(2,1)$ in Example 16. This retailer type, which has high demand and low holding cost ("strong" in both dimensions), would prefer a situation where his cost is known to the supplier to a situation where both his cost and demand are private information since $114.5+257.4<137.4+351.0$ (see Table 12). Kostamis \& Duenyas (2011) demonstrated that more dimensions of IA are not always preferable for the retailer. In particular, they showed that a type who is strong in one dimension but week in the other would sometimes prefer to get screened along his weak dimension. Our observation, however, corresponds to the type that is strong in both dimensions.

Table 9: Demand scenarios in Example 16

\begin{tabular}{cccccc}
\hline$t$ & 1 & 2 & 3 & 4 & 5 \\
\hline \hline$d_{1, t}$ & 47 & 57 & 58 & 47 & 53 \\
$d_{2, t}$ & 55 & 86 & 84 & 85 & 128 \\
\hline
\end{tabular}

Table 10: Cost parameters in Example 16

(a) Cost parameters of the supplier

\begin{tabular}{cccccc}
\hline$t$ & 1 & 2 & 3 & 4 & 5 \\
\hline \hline$h_{t}^{S}$ & 2.4 & 5.8 & 5.1 & 1.4 & 2.5 \\
$K_{t}^{S}$ & 126.3 & 261.2 & 127.6 & 124.3 & 206.8 \\
$p_{t}^{S}$ & 5.7 & 3.0 & 3.0 & 5.4 & 6.6 \\
\hline
\end{tabular}

(b) Cost parameters of the retailer

\begin{tabular}{cccccc}
\hline$t$ & 1 & 2 & 3 & 4 & 5 \\
\hline \hline$s_{t}^{R}$ & 11.2 & 11.2 & 11.2 & 11.2 & 11.2 \\
$K_{t}^{R}$ & 78.0 & 88.0 & 92.0 & 65.0 & 67.0 \\
$p_{t}^{R}$ & 8.9 & 7.1 & 10.0 & 11.4 & 11.5 \\
\hline
\end{tabular}

Table 11: Individual and coordinated optimal procurement plans (IA on demand and cost)

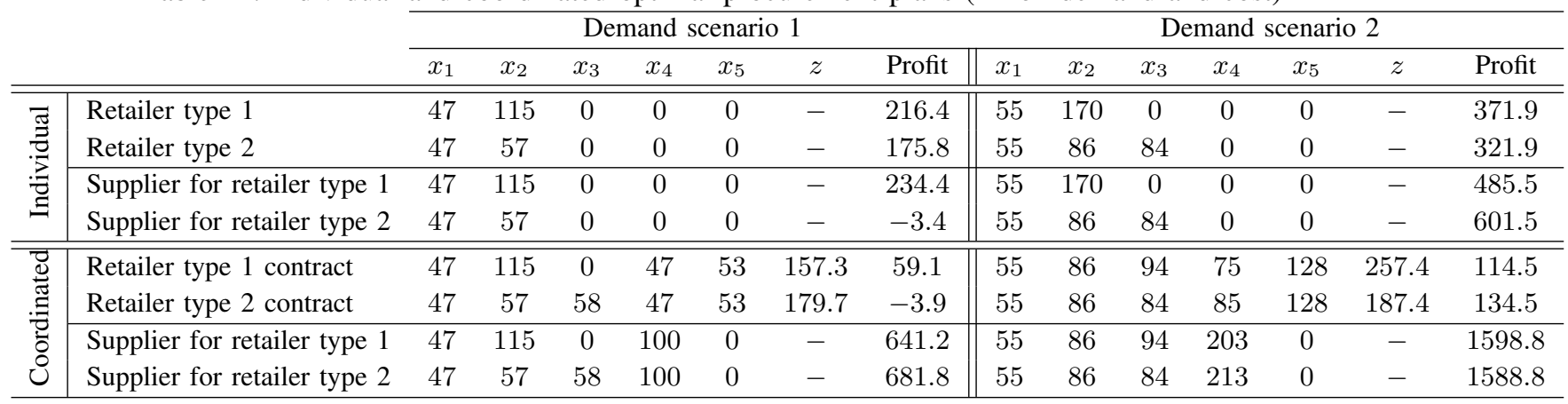

Table 12: Coordinated optimal procurement plans (IA on demand)

\begin{tabular}{lccccccc}
\cline { 2 - 7 } & \multicolumn{7}{c}{ Cost type 1 } \\
\cline { 2 - 8 } & $x_{1}$ & $x_{2}$ & $x_{3}$ & $x_{4}$ & $x_{5}$ & $z$ & Profit \\
\hline \hline Retailer type 1 contract & 47 & 57 & 158 & 0 & 0 & 466.6 & -246.8 \\
Retailer type 2 contract & 47 & 75 & 139 & 0 & 0 & 351.0 & 137.4 \\
\hline
\end{tabular}

\subsubsection{Asymmetric set-up and holding cost information:}

The supplier believes that the retailer can have $M$ set-up cost types with associated probabilities $P_{i}^{d} \geq 0$ for $i \in I=\{1,2, \ldots, M\}$ and $\sum_{i=1}^{M} P_{i}^{d}=1$. The retailer knows his set-up cost with certainty. With the assumption 
that set-up and holding cost types are independent, the supplier's beliefs satisfy $P_{i, j}=\left(P_{i}^{d}\right)\left(P_{j}^{c}\right)$ for $i \in I$, $j \in J$ and $\sum_{i \in I, j \in J} P_{i, j}=1$.

The sequence of events is as follows. At the beginning, the retailer knows both his set-up and holding cost type, unobserved by the supplier. Being aware of the possible cost types, the supplier computes the optimal profits of the retailer for each type $\left(\pi_{i, j}^{R *}\right)$. Then he offers a menu of "procurement plan-side payment" contracts $\left\{\left(x_{i, j, t}\right)_{t \in \mathcal{T}}, z_{i, j}\right\}$ for each pair of assumed retailer types $(i, j)$ such that his expected profit is maximized. The retailer either selects one of the contracts in the menu or imposes his own optimal plan on the supplier.

The supplier's problem of designing the optimal menu can be formulated as follows:

$$
\begin{aligned}
& \max \sum_{i=1}^{M} \sum_{j=1}^{N} P_{i, j}\left(\sum_{t=1}^{T}\left(p_{t}^{R} x_{i, j, t}^{R}-K_{t}^{S} y_{i, j, t}^{S}-p_{t}^{S} x_{i, j, t}^{S}-h_{t}^{S} I_{i, j, t}^{S}\right)-z_{i, j}\right) \\
& \text { s.t. } \quad \pi_{i, j}^{R, i, j}+z_{i, j} \geq \pi_{i, j}^{R *} \\
& \pi_{i, j}^{R, i, j}+z_{i, j} \geq \pi_{i^{\prime}, j^{\prime}}^{R, i, j}+z_{i^{\prime}, j^{\prime}} \\
& \pi_{i^{\prime}, j^{\prime}}^{R, i, j}=\sum_{t=1}^{T}\left(s_{t}^{R} d_{t}-K_{i, t}^{R} y_{i^{\prime}, j^{\prime}, t}^{R}-p_{t}^{R} x_{i^{\prime}, j^{\prime}, t}^{R}-h_{j, t}^{R} I_{i^{\prime}, j^{\prime}, t}^{R, i, j}\right) \\
& I_{i^{\prime}, j^{\prime}, t-1}^{R, i, j}+x_{i^{\prime}, j^{\prime}, t}^{R}=d_{t}+I_{i^{\prime}, j^{\prime}, t}^{R, i, j} \\
& \forall t \in \mathcal{T}, \forall i, i^{\prime} \in I, \forall j, j^{\prime} \in J \\
& x_{i, j, t}^{R} \leq d_{t, T} y_{i, j, t}^{R} \\
& x_{i, j, t}^{R} \geq y_{i, j, t}^{R} \\
& I_{i, j, t-1}^{S}+x_{i, j, t}^{S}=x_{i, j, t}^{R}+I_{i, j, t}^{S} \\
& x_{i, j, t}^{S} \leq d_{t, T} y_{i, j, t}^{S} \\
& x_{i, j, t}^{R} \in \mathbb{Z}_{+} \\
& z_{i, j}, x_{i, j, t}^{S}, I_{i, j, t}^{S}, I_{i^{\prime}, j^{\prime}, t}^{R, i, j} \geq 0 \\
& y_{i, j, t}^{S}, y_{i, j, t}^{R} \in\{0,1\} \\
& I_{i, j, 0}^{S}, I_{i^{\prime}, j^{\prime}, 0}^{R, i, j}=0 \\
& \forall t \in \mathcal{T}, \forall i \in I, \forall j \in J \\
& \forall t \in \mathcal{T}, \forall i \in I, \forall j \in J \\
& \forall t \in \mathcal{T}, \forall i \in I, \forall j \in J \\
& \forall t \in \mathcal{T}, \forall i \in I, \forall j \in J \\
& \forall t \in \mathcal{T}, \forall i \in I, \forall j \in J \\
& \forall t \in \mathcal{T}, \forall i, i^{\prime} \in I, \forall j, j^{\prime} \in J \\
& \forall t \in \mathcal{T}, \forall i \in I, \forall j \in J \\
& \forall i, i^{\prime} \in I, \forall j, j^{\prime} \in J
\end{aligned}
$$

We impose a restriction on the form of the offered contracts: we assume that with stationary cost parameters for both the retailer and supplier, the set of procurement plans satisfy the property of a "consistent contract". While it is challenging to show analytically, the findings from our extensive numerical study indicate that the optimal contracts do satisfy this property. This is also corroborated by the fact that it is an intuitive property to consider for the set of contracts and easy to formalize.

Definition 17. Consistent contracts. Consider the case where the retailer has private information about his setup and holding cost. A contract is consistent if (a) for a fixed set-up cost, the number of set-ups across the whole planning horizon increases as holding cost increases, i.e., for each $K_{i}^{R}$, if $h_{j}^{R}<h_{j^{\prime}}^{R}$ then $\sum y_{i, j, t}^{R} \leq \sum y_{i, j^{\prime}, t}^{R}$; (b) for a fixed holding cost, the total quantity of ending inventories across the whole planning horizon increases as set-up cost increases, i.e., for each $h_{j}^{R}$, if $K_{i}^{R}<K_{i^{\prime}}^{R}$ then $\sum I_{i, j, t}^{R, i, j} \leq \sum I_{i^{\prime}, j, t}^{R, i^{\prime}, j}$.

The consistent contract property can be easily incorporated into the supplier's problem as follows: 


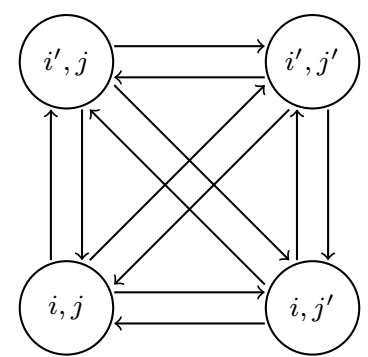

Figure 4: Network of types with two dimensions of private information

$$
\begin{aligned}
\sum_{t=1}^{T} y_{i, j, t}^{R} \leq \sum_{t=1}^{T} y_{i, j^{\prime}, t}^{R} & \forall i \in I, \forall j, j^{\prime} \in J, j<j^{\prime} \\
\sum_{t=1}^{T} I_{i, j, t}^{R, i, j} \leq \sum_{t=1}^{T} I_{i^{\prime}, i^{\prime}, t}^{R, t} & \forall i, i^{\prime} \in I, i<i^{\prime}, \forall j \in J
\end{aligned}
$$

Note that the consideration of consistent contracts does not lead to an empty feasible region as the outside option of each type $(i, j)$ is a feasible solution that satisfies the consistency property (see Van Hoesel \& Wagelmans (2000)). An example of this setting is given in Subsection 7.2.

\subsection{Network interpretation}

In this subsection, we take a closer look at the model with IA on two cost parameters. We discuss the relevant IC constraints and identify the redundant ones. Throughout this subsection we assume that the retailer's private information, i.e., his holding and set-up cost as well as the unit procurement cost are time-invariant.

To structure our analysis, we first classify the set of IC constraints as follows. A retailer type is a pair of $(i, j)$ where $i$ is the set-up cost and $j$ is the holding cost and $K_{i^{\prime}}^{R}>K_{i}^{R}$, while $h_{j^{\prime}}^{R}<h_{j}^{R}$. The set of IC constraints in (72) can be divided into eight sets as follows:

- Vertical upward IC (VUIC) constraints: The retailer type $(i, j)$ chooses the contract designed for type

$$
\left(i^{\prime}, j\right) \text { where } i^{\prime}>i \text { : } \quad \pi_{i, j}^{R, i, j}+z_{i, j} \geq \pi_{i^{\prime}, j}^{R, i, j}+z_{i^{\prime}, j}
$$

- Vertical downward IC (VDIC) constraints: The retailer type $\left(i^{\prime}, j\right)$ chooses the contract designed for type $(i, j)$ where $i^{\prime}>i$ :

$$
\pi_{i^{\prime}, j}^{R, i^{\prime}, j}+z_{i^{\prime}, j} \geq \pi_{i, j}^{R, i^{\prime}, j}+z_{i, j}
$$

- Horizontal downward IC (HDIC) constraints: The retailer type $(i, j)$ chooses the contract designed for type $\left(i, j^{\prime}\right)$ where $j^{\prime}<j$ :

$$
\pi_{i, j}^{R, i, j}+z_{i, j} \geq \pi_{i, j^{\prime}}^{R, i, j}+z_{i, j^{\prime}}
$$

- Horizontal upward IC (HUIC) constraints: The retailer type $\left(i, j^{\prime}\right)$ chooses the contract designed for type $(i, j)$ where $j^{\prime}<j$ :

$$
\pi_{i, j^{\prime}}^{R, i, j^{\prime}}+z_{i, j^{\prime}} \geq \pi_{i, j}^{R, i, j^{\prime}}+z_{i, j}
$$

- Diagonal downward IC (DDIC) constraints: The retailer type $\left(i^{\prime}, j^{\prime}\right)$ chooses the contract designed for type $(i, j)$ where $i^{\prime}>i$ and $j^{\prime}<j: \quad \pi_{i^{\prime}, j^{\prime}}^{R, i^{\prime}, j^{\prime}}+z_{i^{\prime}, j^{\prime}} \geq \pi_{i, j}^{R, i^{\prime}, j^{\prime}}+z_{i, j}$ 
- Diagonal upward IC (DUIC) constraints: The retailer type $(i, j)$ chooses the contract designed for type $\left(i^{\prime}, j^{\prime}\right)$ where $i^{\prime}>i$ and $j^{\prime}<j$ :

$$
\pi_{i, j}^{R, i, j}+z_{i, j} \geq \pi_{i^{\prime}, j^{\prime}}^{R, i, j}+z_{i^{\prime}, j^{\prime}}
$$

- Off-diagonal downward IC (ODDIC) constraints: The retailer type $\left(i^{\prime}, j\right)$ chooses the contract designed for type $\left(i, j^{\prime}\right)$ where $i^{\prime}>i$ and $j^{\prime}<j_{\dot{\pi}_{i^{\prime}, j}^{R, i^{\prime}, j}}^{:}+z_{i^{\prime}, j} \geq \pi_{i, j^{\prime}}^{R, i^{\prime}, j}+z_{i, j^{\prime}}$

- Off-diagonal upward IC (ODUIC) constraints: The retailer type $\left(i, j^{\prime}\right)$ chooses the contract designed for type $\left(i^{\prime}, j\right)$ where $i^{\prime}>i$ and $j^{\prime}<j: \quad \pi_{i, j^{\prime}}^{R, i, j^{\prime}}+z_{i, j^{\prime}} \geq \pi_{i^{\prime}, j}^{R, i, j^{\prime}}+z_{i^{\prime}, j}$

In addition to the previously considered IC constraints in the one-dimensional case, the two-dimensional setting requires us to consider new diagonal IC constraints.

Next, we simplify the set of IC constraints. Theorems 18 and 19 respectively show under which conditions the horizontal and vertical IC constraints are satisfied. The sufficiency of local upward/downward horizontal and vertical IC constraints is shown in Theorem 20.

Theorem 18. A set of procurement plans satisfies HUIC and HDIC constraints iff it is monotonic in the holding cost $(j)$ component.

Proof: With a fixed set-up cost $\left(K_{i}^{R}\right)$ and $h_{j^{\prime}}^{R}<h_{j}^{R}$, consider the non-negative cycle condition applied to cycle $(i, j) \rightarrow\left(i, j^{\prime}\right) \rightarrow(i, j)$ in Figure 4 . Given the assumption of time-invariant procurement, holding and set-up cost, it implies:

$$
\begin{array}{r}
\left(\pi_{i, j^{\prime}}^{R, i, j^{\prime}}-\pi_{i, j}^{R, i, j^{\prime}}\right)+\left(\pi_{i, j}^{R, i, j}-\pi_{i, j^{\prime}}^{R, i, j}\right) \geq 0 \\
\Leftrightarrow \sum_{t=1}^{T}\left(s_{t}^{R} d_{t}-K_{i}^{R} y_{i, j^{\prime}, t}^{R}-p_{t}^{R} x_{i, j^{\prime}, t}^{R}-h_{j^{\prime}}^{R} I_{i, j^{\prime}, t}^{R, i, j^{\prime}}\right)-\sum_{t=1}^{T}\left(s_{t}^{R} d_{t}-K_{i}^{R} y_{i, j, t}^{R}-p_{t}^{R} x_{i, j, t}^{R}-h_{j,}^{R} I_{i, j, t}^{R, i, j^{\prime}}\right) \\
+\sum_{t=1}^{T}\left(s_{t}^{R} d_{t}-K_{i}^{R} y_{i, j, t}^{R}-p_{t}^{R} x_{i, j, t}^{R}-h_{j}^{R} I_{i, j, t}^{R, i, j}\right)-\sum_{t=1}^{T}\left(s_{t}^{R} d_{t}-K_{i}^{R} y_{i, j^{\prime}, t}^{R}-p_{t}^{R} x_{i, j^{\prime}, t}^{R}-h_{j}^{R} I_{i, j^{\prime}, t}^{R, i, j}\right) \geq 0 \\
\Leftrightarrow \sum_{t=1}^{T} h_{j^{\prime}}^{R}\left(I_{i, j, t}^{R, i, j^{\prime}}-I_{i, j^{\prime} t}^{R, i, j^{\prime}}\right)+\sum_{t=1}^{T} h_{j}^{R}\left(I_{i, j^{\prime}, t}^{R, i, j}-I_{i, j, t}^{R, i, j}\right) \geq 0 .
\end{array}
$$

We know that $I_{i, j, t}^{R, i, j^{\prime}}=I_{i, j, t}^{R, i, j}$ and $I_{i, j^{\prime} t}^{R, i, j^{\prime}}=I_{i, j^{\prime}, t}^{R, i, j}$. Therefore, (85) is equivalent to

$$
\sum_{t=1}^{T}\left(h_{j^{\prime}}^{R}-h_{j}^{R}\right)\left(I_{i, j, t}^{R, i, j^{\prime}}-I_{i, j^{\prime} t}^{R, i, j^{\prime}}\right) \geq 0 \Leftrightarrow \sum_{t=1}^{T} I_{i, j, t}^{R, i, j^{\prime}} \leq \sum_{t=1}^{T} I_{i, j^{\prime} t}^{R, i, j^{\prime}}
$$

Theorem 19. A set of procurement plans satisfies VUIC and VDIC constraints iff it is monotonic in the set-up cost (i) component.

Proof: With a fixed holding cost $\left(h_{j}^{R}\right)$ and $K_{i^{\prime}}^{R}>K_{i}^{R}$, consider the non-negative cycle condition applied to cycle $(i, j) \rightarrow\left(i^{\prime}, j\right) \rightarrow(i, j)$ in Figure 4 . With stationary cost parameters for the retailer, it is easy to verify that the theorem holds by substituting in the expression for $\pi_{i, j}^{R, i, j}$ and canceling common terms.

Theorem 20. Out of all the horizontal and vertical downwards constraints, only the local downward constraints 
relating $(i+1, j)$ and $(i, j)$, and relating $(i, j+1)$ and $(i, j)$ matter. Only the local upward constraints relating $(i, j)$ and $(i+1, j)$, and $(i, j)$ and $(i, j+1)$ matter out of all the horizontal and vertical upward constraints.

Proof: Similar to the proof of Theorem 11 in Section 6.

The next theorem shows the redundancy of diagonal IC constraints.

Theorem 21. The upward / downward diagonal IC constraints are implied by the relevant horizontal and vertical IC constraints. More specifically, (a) the DDIC constraints relating type $\left(i^{\prime}, j^{\prime}\right)$ to $(i, j)$ where $K_{i^{\prime}}^{R}>K_{i}^{R}$ and $h_{j^{\prime}}^{R}<h_{j}^{R}:$

$$
\pi_{i^{\prime}, j^{\prime}}^{R, i^{\prime}, j^{\prime}}+z_{i^{\prime}, j^{\prime}} \geq \pi_{i, j}^{R, i^{\prime}, j^{\prime}}+z_{i, j}
$$

are implied by the HUIC and VDIC constraints, and (b) the DUIC constraints relating type $(i, j)$ to $\left(i^{\prime}, j^{\prime}\right)$ where $K_{i^{\prime}}^{R}>K_{i}^{R}$ and $h_{j^{\prime}}^{R}<h_{j}^{R}$ :

$$
\pi_{i, j}^{R, i, j}+z_{i, j} \geq \pi_{i^{\prime}, j^{\prime}}^{R, i, j}+z_{i^{\prime}, j^{\prime}}
$$

are implied by HDIC and VUIC constraints.

Proof: We prove the claim in part (a). The proof of part (b) is similar. There are two sets of HUIC and VDIC constraints that can be considered to prove the claim. We use the HUIC constraint, relating $\left(i, j^{\prime}\right)$ and $(i, j)$, and VDIC constraint, relating $\left(i^{\prime}, j^{\prime}\right)$ and $\left(i, j^{\prime}\right)$. Note that we could have used the other set (VDIC constraint, relating $\left(i^{\prime}, j\right)$ and $(i, j)$ and HUIC constraint, relating $\left(i^{\prime}, j^{\prime}\right)$ and $\left.\left(i^{\prime}, j\right)\right)$ to prove the claim with a similar argument.

Consider the HUIC and VDIC constraints:

$$
\begin{gathered}
\pi_{i, j^{\prime}}^{R, i, j^{\prime}}+z_{i, j^{\prime}} \geq \pi_{i, j}^{R, i, j^{\prime}}+z_{i, j} \\
\pi_{i^{\prime}, j^{\prime}}^{R, i^{\prime}, j^{\prime}}+z_{i^{\prime}, j^{\prime}} \geq \pi_{i, j^{\prime}}^{R, i^{\prime}, j^{\prime}}+z_{i, j^{\prime}} .
\end{gathered}
$$

By adding these two inequalities and rearranging we have:

$$
\pi_{i^{\prime}, j^{\prime}}^{R, i^{\prime}, j^{\prime}}+z_{i^{\prime}, j^{\prime}}-\pi_{i, j}^{R, i^{\prime}, j^{\prime}}-z_{i, j} \geq\left(\pi_{i, j}^{R, i, j^{\prime}}-\pi_{i, j^{\prime}}^{R, i, j^{\prime}}\right)-\left(\pi_{i, j}^{R, i^{\prime}, j^{\prime}}-\pi_{i, j^{\prime}}^{R, i^{\prime}, j^{\prime}}\right) .
$$

After substituting in the expression for $\pi_{i^{\prime}, j^{\prime}}^{R, i^{\prime}, j^{\prime}}$ and canceling common terms, the right-hand-side of (88) is equivalent to

$$
\sum_{t=1}^{T}\left(K_{i}^{R}-K_{i^{\prime}}^{R}\right)\left(y_{i, j^{\prime}, t}^{R}-y_{i, j, t}^{R}\right)
$$

It is a non-negative value under the assumption of consistent contracts and therefore we have

$$
\pi_{i^{\prime}, j^{\prime}}^{R, i^{\prime}, j^{\prime}}+z_{i^{\prime}, j^{\prime}}-\pi_{i, j}^{R, i^{\prime}, j^{\prime}}-z_{i, j} \geq 0,
$$

which is equivalent to (86) and proves the claim.

To summarize, all the global HDIC, HUIC, VDIC, VUIC plus all the (local and global) DUIC and DDIC constraints are not relevant at the optimal solution. Consider for instance the situation where $M=N=4$. To find all the shortest paths from the dummy vertex $(0,0)$ to each vertex and therefore determine the side payment quantities, we only need to consider a subset of 120 IC constraints instead of the full set with 240 constraints. 
Example 22. Suppose that the retailer has private information about his holding and set-up costs. The supplier assumes 2 stationary set-up and holding costs, arising with the same probability: $h_{1}^{R}=9.5, h_{2}^{R}=6.2, K_{1}^{R}=$ 154.7, and $K_{2}^{R}=280.3$. Other cost parameters are $S^{R}=18, P^{R}=7.1, K^{S}=563.2, h^{S}=3.7$, and $P^{S}=8.8$. Demand is given by $d=(37,74,34,79,18)$. Figure 5 illustrates the network of types in this example. For clarity, we only show the binding IR and IC constraints. Minus the length of the shortest path from the dummy type $(0,0)$ to each type is a lower bound for the optimal side payment quantities. Table 13 shows the optimal menu of contracts. Observe that a type which is inefficient in two dimensions (e.g., high set-up and holding cost) may pretend to be efficient in both dimensions and the other way around. It is, however, not profitable for a low set-up cost and high holding cost to pretend to have high set-up and low holding cost and vice versa.

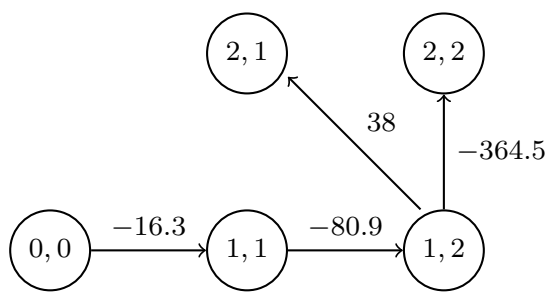

Figure 5: Network of types in Example 22

Table 13: Optimal menu of contracts in Example 22

\begin{tabular}{lcccccccc}
\hline Contract & $x_{1}$ & $x_{2}$ & $x_{3}$ & $x_{4}$ & $x_{5}$ & $z$ & $\pi_{j, j}^{R}$ & $\pi_{j}^{R *}$ \\
\hline \hline Retailer type (1,1) & 37 & 74 & 34 & 97 & 0 & 16.3 & 1848.0 & 1864.3 \\
Retailer type (1,2) & 41 & 104 & 0 & 97 & 0 & 97.2 & 1826.5 & 1907.4 \\
Retailer type (2,1) & 37 & 108 & 0 & 97 & 0 & 59.2 & 1302.9 & 1345.6 \\
Retailer type (2,2) & 145 & 0 & 0 & 97 & 0 & 461.7 & 1085.2 & 1474.5 \\
\hline
\end{tabular}

\section{CONClusion AND FUtuRe RESEARCH}

This paper studies the supplier's problem of eliciting cooperation from a strong retailer who has one or two-dimensional private information about customer demand or his cost parameters. Both the SC members in our analysis are facing a multi-period lot-sizing problem with dynamic and deterministic demand. The supplier uses a simple and easily implementable coordination mechanism, offering a menu of contracts, to influence the retailer's ordering plan and the retailer either accepts one of the contracts or imposes his optimal plan. Each dimension of private information in this study has a discrete type space and the retailer's outside option depends on his private information.

The complexities of a multi-period inventory model, coupled with the intricacies of IA between SC members make the design of optimal supply contracts a nontrivial task. Given the opportunistic reaction of the retailer to the proposed offer, the supplier is facing a nested (bi-level) optimization problem. We have shown that, with a specific assumption on the retailer's cost parameters, this problem can be transformed into a single-level MIP by exploiting the structural properties of optimal solutions at the lower level (retailer) problem. 
Assuming a discrete type space, as opposed to the common binary space in the literature, for the retailer's private information, we presented a network interpretation for the set of incentive compatibility constraints that allows us to analyze the problem from an intuitive graph theoretic viewpoint. In particular, we have shown that a bound for the side payment quantities can be found by considering a shortest path tree which consists of the participation and local incentive constraints. This implies that with a considerably simpler set of incentive constraints, in terms of the number of relevant constraints, we can find the optimal side payment quantities.

A surprising finding in our study is related to the possible reaction of an opportunistic retailer. Following a sensible and well-known result in the one-dimensional mechanism design literature, one can expect that it is in the interest of an efficient (e.g., with a lower cost) retailer type to pretend to be inefficient (e.g., a higher cost) and not the other way around. This implies that the supplier's problem becomes easier as he only needs to consider the efficient type's incentive constraints in order to find the optimal contract menu. However, we showed that it is possible to have an optimal solution in which the supplier gives up some strictly positive information rent to the inefficient type and zero rent to the efficient one. This unexpected result can be attributed to the consideration of the more realistic and fundamentally different assumption of type-dependent outside options.

In the two-dimensional cost IA and considering a binary type space for each dimension with equal probabilities, we showed that a type that is inefficient in two dimensions (e.g., high set-up and holding cost) may pretend to be efficient in both dimensions and the other way around. It is, however, not profitable for a low set-up cost and high holding cost to pretend to have high set-up and low holding cost and vice versa. It was previously shown in the literature that a retailer who is strong in one dimension (e.g., high demand) but weak (e.g., high cost) in the other would sometimes prefer a situation where his weak dimension is known to the supplier to a situation where both dimensions are private information. With an example, we demonstrated that it might be in the interest of a retailer type who is strong in both dimensions that one of the private dimensions is known to the supplier.

This paper focused on the analysis of a coordination problem with two dimensions of private information and a discrete type space for each dimension. A natural next step would be to consider more types (i.e., assume a continuous type space per dimension). Increasing the number of types, however, does not lead to uncovering new results of qualitative significance. In fact, the complexities of the coordination setting and models in place have already made it hardly possible to draw more results for a discrete type space. This limitation is also pointed out by Çakanyıldırım et al. (2012) and Fudenberg \& Tirole (1991), asserting that the problem with more than two types and type-dependent outside options is analytically difficult and does not lead to general results. Another more interesting extension to our study would be to consider more dimensions of private information for the retailer. For instance, our type-dependent outside option assumption is an important step towards enhancing our understanding of a situation which comes closer to reality. This understanding can be taken to the next level by assuming that the retailer possesses private information about his outside option.

Regarding the number of contracts in the offered menu, the revelation principal says that the supplier needs to 
offer a contract for each retailer type. We are in the process of designing the so-called "pooling" or "bunching" contracts where each contract is intended to appeal to more than one type of retailer. This is specifically useful when there are a large variety of retailer types.

The supplier's coordination mechanism in this study, i.e., making a side payment, can be easily modeled as a profit-sharing or an all-unit discount mechanism. It would be interesting to evaluate the performance of other coordination mechanisms such as different rebate and holding cost compensation schemes. Coordination in more complicated settings including a SC with more tiers and more players at each tier can be considered as another direction for extending the current study.

\section{APPENDIX}

Example 23. In this example, we show the necessity of constraint (32). Suppose that the retailer has two set-up cost types, $K_{1, t}^{R}=K_{1}^{R}=282$ and $K_{2, t}^{R}=K_{2}^{R}=73$ arising with the same probability. The supplier's cost parameters are the same as the ones in Table $2 \mathrm{~b}$ and other cost parameters of the retailer are given in Table 14 . The optimal solutions of the supplier's problem described in (26) to (38) with and without constraint (32) are shown in Table 15. Without the constraint, in the procurement plan of type 2 retailer in period 4 , we have $x_{2,4}^{R}=0$ but $y_{2,4}^{R}=1$. This corresponds to a practically infeasible case where the retailer does not order but has to pay a set-up cost in that period.

Table 14: Parameters of the retailer's problem in Example 23

\begin{tabular}{cccccc}
\hline$t$ & 1 & 2 & 3 & 4 & 5 \\
\hline \hline$d_{t}$ & 26 & 25 & 29 & 33 & 27 \\
$h_{t}^{R}$ & 10 & 16 & 10 & 15 & 13 \\
$p_{t}^{R}$ & 15 & 17 & 11 & 17 & 15 \\
$s_{t}^{R}$ & 30 & 30 & 30 & 30 & 30 \\
\hline
\end{tabular}

Table 15: Optimal menu of contracts in Example 23

\begin{tabular}{|c|c|c|c|c|c|c|c|c|}
\hline \multirow[b]{3}{*}{$t$} & \multicolumn{4}{|c|}{ Retailer type 1} & \multicolumn{4}{|c|}{ Retailer type 2} \\
\hline & \multicolumn{2}{|c|}{ with (32) } & \multicolumn{2}{|c|}{ without (32) } & \multicolumn{2}{|c|}{ with (32) } & \multicolumn{2}{|c|}{ without (32) } \\
\hline & $x_{1, t}^{R}$ & $y_{1, t}^{R}$ & $x_{1, t}^{R}$ & $y_{1, t}^{R}$ & $x_{2, t}^{R}$ & $y_{2, t}^{R}$ & $x_{2, t}^{R}$ & $y_{2, t}^{R}$ \\
\hline 1 & 51 & 1 & 51 & 1 & 50 & 1 & 51 & 1 \\
\hline 2 & 0 & 0 & 0 & 0 & 1 & 1 & 0 & 0 \\
\hline 3 & 62 & 1 & 62 & 1 & 62 & 1 & 62 & 1 \\
\hline 4 & 0 & 0 & 0 & 0 & 0 & 0 & 0 & 1 \\
\hline 5 & 27 & 1 & 27 & 1 & 27 & 1 & 27 & 1 \\
\hline$z$ & \multicolumn{2}{|c|}{0} & \multicolumn{2}{|c|}{0} & \multicolumn{2}{|c|}{251} & \multicolumn{2}{|c|}{259} \\
\hline$\pi_{j, j}^{R}$ & \multicolumn{2}{|c|}{922} & \multicolumn{2}{|c|}{922} & \multicolumn{2}{|c|}{1484} & \multicolumn{2}{|c|}{1476} \\
\hline$\pi_{j}^{R *}$ & \multicolumn{2}{|c|}{922} & \multicolumn{2}{|c|}{922} & \multicolumn{2}{|c|}{1735} & \multicolumn{2}{|c|}{1735} \\
\hline
\end{tabular}

\section{REFERENCES}

Aksen, D., Altinkemer, K. \& Chand, S. (2003), 'The single-item lot-sizing problem with immediate lost sales',

European Journal of Operational Research 147(3), 558 - 566.

Bloom, P. N. \& Perry, V. G. (2001), 'Retailer power and supplier welfare: The case of wal-mart', Journal of Retailing 77(3), 379 - 396. 
Çakanyıldırım, M., Feng, Q., Gan, X. \& Sethi, S. (2012), 'Contracting and coordination under asymmetric production cost information', Production and Operations Management 21(2), 345-360.

Chen, F. (2003), Information sharing and supply chain coordination, in S. Graves \& A. de Kok, eds, 'Supply Chain Management: Design, Coordination and Operation', 1th edn, Vol. 11 of Handbooks in Operations Research and Management Science, Elsevier, pp. 341 - 421.

Corbett, C. J. \& de Groote, X. (2000), 'A supplier's optimal quantity discount policy under asymmetric information', Management Science 46(3), pp. 444-450.

Dudek, G. \& Stadtler, H. (2005), 'Negotiation-based collaborative planning between supply chains partners', European Journal of Operational Research 163(3), 668-687.

Dudek, G. \& Stadtler, H. (2007), 'Negotiation-based collaborative planning in divergent two-tier supply chains', International Journal of Production Research 45(2), 465-484.

Fudenberg, D. \& Tirole, J. (1991), Game Theory, MIT Press.

Ha, A. Y. (2001), 'Supplier-buyer contracting: Asymmetric cost information and cutoff level policy for buyer participation', Naval Research Logistics 48(1), 41-64.

Kostamis, D. \& Duenyas, I. (2011), 'Purchasing under asymmetric demand and cost information: When is more private information better?', Operations Research 59(4), 914-928.

Laffont, J. \& Martimort, D. (2001), The theory of incentives: the principal-agent model, Princeton University Press.

Muthoo, A. (1999), Bargaining theory with applications, Cambridge University Press, Cambridge, UK.

Myerson, R. B. (1981), 'Optimal auction design', Mathematics of Operations Research 6(1), 58-63.

Özer, O. \& Wei, W. (2006), 'Strategic commitments for an optimal capacity decision under asymmetric forecast information', Management Science 52(8), 1238-1257.

Perakis, G. \& Roels, G. (2007), 'The price of anarchy in supply chains: Quantifying the efficiency of price-only contracts', Management Science 53(8), 1249-1268.

Rochet, J. (1987), 'A necessary and sufficient condition for rationalizability in a quasi-linear context', Journal of Mathematical Economics 16(2), 191 - 200.

Sucky, E. (2006), 'A bargaining model with asymmetric information for a single supplier-single buyer problem', European Journal of Operational Research 171(2), 516 - 535.

Taylor, T. A. (2006), 'Sale timing in a supply chain: When to sell to the retailer', Manufacturing \& Service Operations Management 8(1), 23-42.

Van Hoesel, C. \& Wagelmans, A. (2000), 'Parametric analysis of setup cost in the economic lot-sizing model without speculative motives', International Journal of Production Economics 66(1), 13 - 22.

Vohra, R. (2011), Mechanism Design: a linear programming approach, Cambridge University Press.

Zangwill, W. I. (1969), 'A backlogging model and a multi-echelon model of a dynamic economic lot size production system-a network approach', Management Science 15(9), pp. 506-527. 
Zhang, F. (2010), 'Procurement mechanism design in a two-echelon inventory system with price-sensitive demand', Manufacturing \& Service Operations Management 12(4), 608-626. 